Publisher: Taylor \& Francis \& Informa UK Limited, trading as Taylor \& Francis Group

Journal: Expert Review of Vaccines

DOI: $10.1080 / 14760584.2021 .1874923$

\title{
Plasmodium infection and drug cure for malaria vaccine development
}

Reshma J. Nevagi, ${ }^{1}$ Michael F. Good, ${ }^{1}$ and Danielle I. Stanisic ${ }^{1 *}$

${ }^{1}$ Institute for Glycomics, Griffith University, Gold Coast Campus, Southport, QLD 4215,

Australia

${ }^{*}$ Corresponding Author: Dr Danielle I. Stanisic, PhD, Institute for Glycomics, Griffith University, Gold Coast Campus, Southport, QLD 4215, Australia.

Phone number: +617 55529433

Email: d.stanisic@griffith.edu.au

Funding: This manuscript has been funded by National Health and Medical Research Council (Australia) grants.

Declaration of interest: M F Good has received grants from the National Health and Medical Research Council (Australia). The authors have no other relevant affiliations or financial involvement with any organization or entity with a financial interest in or financial conflict with the subject matter or materials discussed in the manuscript apart from those disclosed.

Reviewer Disclosures: Peer reviewers on this manuscript have no relevant financial or other relationships to disclose. 
Author Contributions: All authors contributed to the writing and revision of this manuscript.

\begin{abstract}
Introduction: Despite decades of research into the development of a vaccine to combat the malaria parasite, a highly efficacious malaria vaccine is not yet available. Different whole parasite-based vaccine approaches, including deliberate Plasmodium infection and drug cure (IDC), have been evaluated in pre-clinical and early phase clinical trials. The advantage of whole parasite vaccines is that they induce immune responses against multiple parasite antigens, thus lowering the impact of antigenic diversity. Deliberate Plasmodium IDC, as a vaccine approach, involves administering infectious, live parasites in combination with an anti-malarial drug, which controls the infection and enables induction of protective immune responses.
\end{abstract}

Areas covered: We describe the evaluation of the Plasmodium IDC approach, using sporozoites or asexual blood-stage parasites in pre-clinical malaria models and in clinical studies in malaria-naïve individuals.

Expert Opinion: Studies in malaria-naïve volunteers have demonstrated that sporozoite IDC approaches can induce superior sterile homologous protective immunity; further optimization may augment the strain-transcending nature of this protective immune response to enable strong protection against heterologous strains. There has been less progress with asexual blood-stage IDC however, pre-clinical and clinical data demonstrate induction of protective 
immunity. The IDC whole parasite approach is extremely promising for development of an effective malaria vaccine.

Keywords: Anti-malarial drugs, Controlled human infection, Immunity, Malaria, Malaria vaccine, Controlled Infection Immunization (CII), Chemoprophylaxis with sporozoites (CPS), Chemoprophylaxis vaccination (CVac).

\section{Article Highlights}

- Malaria is a major global public health threat, causing severe illness and death particularly in children $<5$ yrs of age. A highly effective malaria vaccine is urgently needed.

- Deliberate Plasmodium infection and drug cure (IDC), as a malaria vaccine approach, involves administering infectious, live parasites in combination with an anti-malarial drug, which controls the infection and enables induction of protective immune responses.

- The Plasmodium IDC vaccine approach is being developed using sporozoites or asexual blood-stage parasites contained within red blood cells. They target the sporozoite/liver-stage and blood-stage parasites respectively.

- The sporozoite approaches (chemoprophylaxis with sporozoite immunization by mosquito bite (CPS), and chemoprophylaxis vaccination with purified sporozoites administered by injection (CVac)) induce strong sterile protection against challenge with homologous sporozoites both in rodent models of malaria and in malaria-naïve individuals. Further optimization may induce equivalent levels of protective immunity against heterologous parasites. Route of administration, sporozoite dose, choice and 
timing of administration of anti-malarial drug are all critical variables for the induction of protective immunity.

- Progress with the asexual blood-stage parasite vaccine approach (Controlled infection immunization (CII)) is behind that of the sporozoite vaccine approaches, however the existing pre-clinical and clinical data demonstrate induction of strong homologous protective immunity. For blood-stage CII, parasite persistence following immunization is critical for the induction of protective immunity. Additional studies will be needed to evaluate the protective efficacy of the blood-stage CII vaccine approach against homologous and heterologous parasites in clinical studies.

- Further development and optimization of the Plasmodium IDC approaches are required for the development of a highly effective malaria vaccine; however, it remains a promising vaccine strategy for the induction of a broad protective immunity. 


\subsection{Introduction}

Malaria is a major global public health threat, causing severe illness and death particularly in children $<5$ yrs of age. In 2018, there were an estimated 228 million clinical cases and 405,000 deaths attributable to infection with the malaria parasite [1]. Nearly $85 \%$ of global malaria deaths in 2018 occurred in 19 countries of the African region and India [1].

\subsection{Life-cycle}

Malaria is transmitted to humans through the bite of a Plasmodium spp--infected female Anopheles mosquito. During a bloodmeal, sporozoites in the salivary glands of the mosquito are transferred into the dermis of human skin (Figure 1) [2]. They then migrate to the liver via the bloodstream, infect hepatocytes and mature into hepatic schizonts. $P$. vivax and $P$. ovale have dormant liver-stage parasites (hypnozoites) that may reactivate, leading to relapses months or even years after the recovery of patients from the first episode of malaria. Upon rupture of an infected hepatic schizont, tens of thousands of merozoites are released into the bloodstream where they invade erythrocytes and continue to develop through the different erythrocytic stages (rings, trophozoites and schizonts), before the erythrocyte ruptures to release daughter merozoites. These merozoites also invade erythrocytes and the ongoing cycles of asexual replication enable parasite growth in the blood. During each replication 
cycle, some of the parasites develop into sexual-stage gametocytes which are ingested by mosquitoes during a bloodmeal. In the mosquito, male and female gametocytes mate within the gut forming diploid zygotes, which then become ookinetes. The ookinetes migrate to the midgut of the mosquito, pass through the gut wall and form oocysts. Sporozoites are formed after meiotic division of the oocysts. Sporozoites migrate to the salivary glands of the female Anopheles mosquito, ready to transmit the infection to another human host and continue the cycle of transmission.

\subsection{Current control methods for the malaria parasite}

The current methods of malaria prevention and vector control, such as the use of insecticidetreated nets, indoor residual spraying and preventive therapies for pregnant women and infants, have been effective in significantly reducing the number of malaria cases over the last decade [1]. However, limitations such as the development of resistance against insecticides by mosquitoes [3], environmental changes and development of resistance against existing anti-malarial drugs by the parasite [4] dictate an urgent need to identify additional methods for the prevention and eradication of malaria.

An efficacious vaccine will be the most effective means of preventing infection with the malaria parasite. Two approaches currently being investigated for the development of a malaria vaccine are: sub-unit vaccines containing single or multiple recombinant malaria antigens and whole parasite vaccines containing killed or attenuated (e.g. chemical, irradiated or genetic) whole malaria parasites. Sub-unit vaccines, including the most advanced malaria vaccine Mosquirix $^{\mathrm{TM}}$, demonstrated limited efficacy when evaluated in residents of malariaendemic regions [5-9]. This is thought to be due in large part to heterogeneity in the vaccine antigens and inadequate antibody and memory responses. Whole parasite vaccines are a feasible alternative as this approach maximises the number of antigens included in the 
vaccine, reducing the impact of antigenic variation and primarily inducing a cellular immune response, which targets both surface and intracellular antigens [10]. There are a number of whole parasite vaccine candidates currently in development, containing either whole sporozoites (to target the pre-erythrocytic stage) or parasitized red blood cells (pRBCs) to target the erythrocytic stage.

\subsection{Plasmodium infection and drug cure as a vaccine approach}

One approach for the development of a whole parasite vaccine involves inducing protective immunity by infecting individuals with viable sporozoites or asexual blood-stage parasites and curtailing the infection with drug treatment. For sporozoite vaccine approaches, the antimalarial drug treatment targets liver-stage and/or blood-stage parasites, whereas for asexual blood-stage vaccines, the drug treatment targets the blood-stage parasites only. This exposes the recipients to very low-level blood-stage infections (reviewed in [11]). Multiple cycles of infection and drug treatment may be required to generate optimal protective immunity.

Historically, deliberate infection of humans with Plasmodium parasites has been employed for a number of reasons including as a treatment (malariotherapy) for neurosyphilis in the 1920-1960s [12,13] and to test novel anti-malarial drugs and vaccines (reviewed in [11]). Malariotherapy relied on the malaria parasites inducing fever which is thought to kill the Treponema pallidum organisms. Retrospective analyses of the malariotherapy studies suggest that the observed decrease in parasitemia and febrile episodes during second infections with the same or different strain/species of Plasmodium reflected the induction of partial clinical and/or anti-parasite immunity [14]. Together with observations regarding the development of clinical immunity following multiple infections in individuals living in malaria endemic areas (reviewed in [15]), these data support developing deliberate, controlled malaria infection as a vaccine approach. 
This Plasmodium infection and drug cure (IDC) approach is based on the in vivo inactivation of live, whole Plasmodium spp. parasites via concurrent or subsequent administration of an anti-malarial drug, in a controlled manner over a stipulated time (reviewed in [11]). This review will discuss immunization with sporozoites and blood-stage Plasmodium spp. parasites, combined with the administration of anti-malarial drugs prophylactically or as a treatment, as a vaccination approach (Figure 2a) (Table 1).

\subsection{Plasmodium infection and drug cure with sporozoites}

In 1976, the protocol for in vitro culture of malaria parasites was published by Trager and Jensen [16]. This significantly advanced malaria research by providing an in vitro process for the cultivation of blood-stage parasites and for generating Plasmodium-infected mosquitoes using cultured gametocytes. The first controlled infection with $P$. falciparum, via the bites of sporozoite-infected mosquitoes fed on laboratory cultures. was trialled in 1986 in healthy human volunteers in the USA [17]. More recently, cryopreserved, vialed sporozoites (Sanaria ${ }^{\circledR}$ PfSPZ Challenge) have also become available, which allows infection with a precise number of sporozoites via parenteral injection [18].

Plasmodium IDC with sporozoites entails multiple immunizations by either the bites of laboratory reared Plasmodium-infected mosquitoes or direct injection of purified sporozoites via needle and syringe, in combination with an anti-malarial drug. Chloroquine (CQ) has been widely utilized with sporozoites as it allows complete intra-hepatic parasite development and stops the development of blood-stage parasites [19]. Other drugs, some of which act at different points in the Plasmodium life-cycle, such as mefloquine (MQ)[20], azithromycin (Az) [21], artesunate [22], isopentaquine [23], arteether [24], piperaquine (PPQ) 
[25], primaquine (PQ) [26] and pyrimethamine (PYR) [27] have also been investigated in the context of Plasmodium infection and drug cure with sporozoites (Figure 2b). The use of PQ in any clinical Plasmodium IDC regimen would entail additional safety considerations due to the risk of dose-dependent PQ-induced hemolysis in glucose-6-phosphate (G-6-PD) deficient individuals [28]. Vaccine efficacy is usually determined by whether patent blood-stage parasitemia develops following parasite challenge. Where blood-stage infection does develop, other endpoints such as reduction in liver parasite load can also be examined. In human studies, blood-stage parasitemia data generated by sensitive qPCR methods can be applied into mathematical models to estimate liver parasite burden [29,30].

\subsection{Pre-clinical evaluation of Plasmodium infection and drug cure with sporozoites}

\subsubsection{P. berghei sporozoite infection and drug cure}

\subsubsection{Protection induced by P. berghei sporozoite infection and drug cure}

Sporozoite infection under CQ cover was first studied with $P$. berghei and demonstrated the induction of stage-specific immunity against homologous sporozoite challenge [31]. Mice immunized with $P$. berghei sporozoites and CQ received an intravenous (i.v.) injection of $5 \mathrm{x}$ $10^{4}$ P. berghei sporozoites (five doses, two weeks apart) while receiving CQ during the entire immunization period. Low level blood-stage parasitemias were observed following each immunization. Following the last injection of sporozoites, a 10-day curative course of PQ was given concurrently with CQ to eradicate any remaining parasites from the liver prior to sporozoite challenge. All sporozoite + CQ immunized mice survived challenge, whereas all control mice succumbed to the infection [31]. To examine the stage-specificity of the immune response, immunized mice that survived the sporozoite challenge were re-challenged with pRBCs; however, they did not survive despite being exposed to low level blood-stage infection during the immunization period. This indicates that protection was primarily 
mediated by pre-erythrocytic immunity. This was the first study to demonstrate that immunization with normal infective sporozoites in combination with suppressive doses of an anti-malarial drug (CQ) could provide protection against sporozoite challenge comparable to that observed with another leading pre-erythrocytic vaccine candidate, radiation attenuated sporozoites (RAS) [32,33]. A further comparative study established that one or two i.v. injections of $10^{4} \mathrm{P}$. berghei sporozoites $+\mathrm{CQ}$ induced protection against homologous sporozoite challenge in $57 \%$ and $>96 \%$ of immunized mice respectively, which was similar to what was observed in mice immunized with one or two i.v. doses of $10^{4}$ RAS [34].

It has been suggested that the protective efficacy of the sporozoite infection $+\mathrm{CQ}$ regimen may not just reflect its ability to enable induction of protective immunity through exposure to a low level controlled malaria infection, it may also be due to the immunomodulatory effects of CQ [35]. In vitro cross-presentation of soluble viral antigens by DCs is enhanced in the presence of CQ and treatment of mice with CQ has been shown to improve $\mathrm{CD} 8^{+} \mathrm{T}$-cell responses to soluble malaria parasite antigen immunization in vivo [36]. The immunomodulatory activity of CQ was therefore investigated in the context of the P. berghei sporozoite IDC regimen by directly comparing IDC with CQ or MQ (whose immunomodulatory effects do not include enhancing cross-presentation) [20]. Both drugs arrest early blood-stage parasites without an effect on pre-erythrocytic parasite stages. Mice received three i.v. doses of $2 \times 10^{4}$ sporozoites at weekly intervals with CQ or MQ. This study did not investigate a direct effect of CQ on cross-presentation in vitro; however, parasite-specific $\mathrm{CD}^{+}$T-cell responses and protection from sporozoite challenge were assessed. Replacement of CQ with MQ led to a significant reduction in the percentage of IFN- $\gamma$ producing memory $\mathrm{CD}^{+} \mathrm{T}$-cells in the liver, but similar protective efficacy against challenge was observed for both the CQ and MQ regimens. Thus, the study provided no 
indication of a direct beneficial effect of CQ on the efficacy of the P. berghei sporozoite + CQ regimen.

Additional anti-malarial drugs have been examined in the context of the sporozoite IDC approach using the $P$. berghei model. Piperaquine is structurally related to CQ and has a prolonged elimination half-life; therefore, it was explored in the context of sporozoite IDC for single-dose chemoprophylaxis, where it is administered at the time of sporozoite injection [25]. Although a single i.v. immunization of C57BL/6 mice with $5 \times 10^{4}$ sporozoites and PPQ resulted in prolonged pre-patency compared to drug-only controls following challenge, all mice succumbed to blood-stage infection. In contrast, two i.v. immunizations with $10^{4} P$. berghei sporozoites and PPQ (4 weeks apart) induced complete sterile protection postchallenge, with an expansion of effector memory CD8 ${ }^{+}$T-cells in the liver and spleen. Induction of complete protective immunity, requiring only a single dose of anti-malarial drug at the time of sporozoite inoculation, instead of multiple doses throughout the immunization period, is a significant advance for a human sporozoite vaccine based on the IDC approach.

Sporozoite IDC with Az has also been examined. Az allows complete intrahepatic development of the parasite and through its delayed death mechanism-of-action and abrogation of apicoplast function, permits the emergence of non-infectious liver-stage merozoites [37]. Two immunizations of $10^{4} \mathrm{P}$. berghei sporozoites $+\mathrm{Az}$ induced sterile protection against homologous sporozoite challenge in $80 \%$ of the mice, which was significantly higher than mice that received two immunizations with either sporozoites $+\mathrm{CQ}$ or RAS [21]. The time to development of parasitemia in mice immunized with sporozoites + $\mathrm{Az}$ and control mice following blood-stage challenge were identical, indicating that the protection induced by the sporozoite + Az regimen was attributable to pre-erythrocytic immunity. 
An attenuated infection, enabled by the sporozoite IDC approach, was also examined in the context of modulating blood-stage malaria infection and severe malaria disease progression in a $P$. berghei model of experimental cerebral malaria (ECM). Sub-therapeutic doses of isopentaquine were administered prior to a single i.v. administration of $10^{4} \mathrm{P}$. berghei sporozoites [23]. Isopentaquine has comparable liver-stage mode-of-action to PQ. Mice were monitored following this immunization; the immunized mice had a delayed patency in parasitemia of 3-4 days compared to sporozoite-only injected control mice and did not show any typical cerebral symptoms. Nevertheless, the immunized mice died 25-30 days postinfection due to hyperparasitemia-associated anaemia. Interestingly, the number of liver-stage parasites did not differ in protected animals, but the parasites were considerably less developed. The $P$. berghei sporozoite + isopentaquine regimen was associated with stronger proinflammatory responses at earlier time points compared with control mice who developed ECM. This included increased T-cell activation in the liver and spleen, increased numbers of effector T-cells, particularly $\mathrm{CD}^{+}$T-effector cells, increased IFN- $\gamma, \mathrm{TNF}-\alpha$, and IL-2 production by $\mathrm{CD} 8^{+} \mathrm{T}$-cells. This was followed by subsequent downregulation of the Th1 response later in the infection, possibly mediated by IL-10. Adoptive transfer and cell depletion studies indicated that a sub-population of $\mathrm{CD}^{+} \mathrm{T}$-cells was responsible for protection observed in this model. This study suggested that attenuating the parasite infection at the liver-stage using drugs such as isopentaquine can modify immunopathogenesis and prevent development of cerebral malaria.

The potential immunomodulatory effect of a concurrent blood-stage infection on the induction of immunity induced by a pre-erythrocytic malaria vaccine is a consideration for eventual application in malaria-endemic regions. In a previous study using $P$. yoelii, a bloodstage infection was shown to suppress both existing $\mathrm{CD} 8^{+} \mathrm{T}$-cell responses against a liver stage-antigen and inhibit initiation of new responses [38]. This phenomenon was also 
examined in the context of the P. berghei sporozoite + CQ regimen to determine the effect of a concurrent Plasmodium blood-stage infection on the induction of pre-erythrocytic immunity [39]. A modified P. berghei sporozoite + CQ immunization schedule was established with $10^{6} \mathrm{pRBCs}$ administered four days prior to each sporozoite immunization ( $10^{4}$ sporozoites); the CQ administration regimen allowed 5 days of blood-stage parasite positivity following each immunization. The presence of blood-stage parasites did not negatively impact on protective immunity induced by a three dose $P$. berghei sporozoite $+\mathrm{CQ}$ immunization regimen, as immunized mice showed complete protection following sporozoite challenge. Although rodent studies do not necessarily predict what will occur in humans, these results are of particular interest in relation to the requirement for treatment of malaria infections in malaria endemic areas prior to administering a malaria vaccine. Drug treatment of existing blood-stage infections may be required for optimal induction of vaccine-induced responses. It would however, present additional complexities for vaccination in a malaria endemic area, both practically for the administration of anti-malarial treatment and for the subsequent timing of the vaccination due to the potential impact of the anti-malarial drugs on the live parasites administered in the vaccine.

\subsubsection{Immune correlates of protection for P. berghei sporozoite infection and drug cure}

Immune correlates of protection for the $P$. berghei sporozoite + CQ immunization regimen were examined in different studies $[39,40]$. Complete protection in $P$. berghei sporozoite + CQ immunized C57BL/6 mice was dependent on intrahepatic IFN- $\gamma$ producing $\mathrm{CD}^{+}$ memory T-cells [40]. Changes to memory $\mathrm{CD}^{+} \mathrm{T}$-cells in immunized mice were minimal. These data suggest that the IFN- $\gamma$ response may be useful as a predictor of the longevity of immunity. Importantly, the decreasing $\mathrm{CD} 8^{+} \mathrm{T}$-cell response was boosted by re-exposure to wild-type sporozoites. Similarly, in another study, protection induced by $P$. berghei sporozoite $+\mathrm{CQ}$ immunization regimen was shown to be mediated by $\mathrm{CD} 8^{+} \mathrm{T}$-cells, with 
depletion of $\mathrm{CD}^{+}$T-cells immediately prior to sporozoite challenge resulting in all $P$. berghei sporozoite + CQ immunized C57BL/6 mice succumbing to challenge infection [39]. There was, however, a delay in the onset of patency in these $\mathrm{CD} 8^{+} \mathrm{T}$-cell depleted mice compared to naïve controls, suggesting that there was residual protective immunity in the absence of $\mathrm{CD}^{+} \mathrm{T}$-cells which may indicate a limited role for other components of the immune system. Adoptive transfer of splenocytes from $P$. berghei sporozoite $+\mathrm{CQ}$ immunized mice into naïve mice, also resulted in complete protection against sporozoite challenge.

It has been suggested that a combination of cellular immunity $\left(\mathrm{CD} 8^{+} \mathrm{T}\right.$-cells $)$ and antibodies is responsible for protection induced by P. berghei sporozoite + CQ immunization [41]. In a passive transfer study, protection was observed in $90 \%$ of C57BL/6 mice transfused prior to challenge with a mixture of splenocytes and immune serum derived from $P$. berghei sporozoite + CQ immunized mice [41]. In contrast, protection against sporozoite challenge was observed in only $50 \%$ and $60 \%$ of C57BL/6 mice that received either splenocytes or immune serum respectively from $P$. berghei sporozoite + CQ immunized mice. This suggests that there is an additive protective effect of cellular and antibody-mediated immunity. To further investigate the role of cellular immunity, C57BL/6 $\mathrm{MHC}$ I $\mathrm{KO}\left(\mathrm{CD}^{+} \mathrm{T}\right.$-cell deficient) or MHC II KO (CD4 ${ }^{+}$T-cell deficient) mice were immunized with the sporozoite + $\mathrm{CQ}$ regimen and compared with C57BL/6 wild-type mice. Following challenge, the immunodeficient mice were only partially protected $(50-60 \%$ did not develop blood-stage parasitemia) whereas C57/BL6 wild-type mice were completely protected. These data suggest a role for both $\mathrm{CD}^{+}$and $\mathrm{CD}^{+} \mathrm{T}$-cells. The presence of circulating $\mathrm{CD} 8^{+}$, killer-cell lectin like receptor G1 $(\mathrm{KLRG} 1)^{\text {high }}, \mathrm{CD} 27^{\text {low }}, \mathrm{CD} 44^{\text {high }}, \mathrm{CD} 62 \mathrm{~L}^{\text {low }}$ cells was shown to be associated with protection following $P$. berghei sporozoite + CQ immunization, warranting further investigation as an immune correlate of protection [41]. KLRG1 is a co-inhibitory 
receptor that is expressed on NK-cells and memory $\mathrm{CD}^{+}$and $\mathrm{CD} 8^{+} \mathrm{T}$-cells and is frequently used as a marker of cellular differentiation and T-cell memory.

\subsubsection{Factors affecting induction of protective immunity by $P$. berghei sporozoite} infection and drug cure: route of administration

Previous studies using the RAS vaccine demonstrated that route of immunization can significantly affect immunogenicity and efficacy both in rodents [42] and humans [43]. Thus, examining this in the context of sporozoite IDC is important.

Mice immunized via an intradermal (i.d.) route with $P$. berghei sporozoites + CQ had reduced protection against sporozoite challenge [44]. This reduction was associated with a 30 -fold lower parasite liver load in the mice who received their vaccination i.d. compared with the mice who were vaccinated via the i.v. route. Intradermal immunization with sporozoites, unlike i.v. immunization, failed to result in the expansion of effector $\mathrm{CD} 8^{+}$ memory T-cells and led to decreased sporozoite-specific $\mathrm{CD} 8^{+}$IFN- $\gamma$ responses in the liver and spleen. These data highlight the importance of optimization of the vaccination regimen to achieve maximal protection. Increasing the number of sporozoites administered i.d. may significantly improve protective efficacy, as was observed with the P. yoelii RAS vaccination model.

\subsubsection{P. yoelii sporozoite infection and drug cure}

\subsubsection{Protection induced by $P$. yoelii sporozoite infection and drug cure}

$P$. yoelii sporozoite IDC was examined with different anti-malarial drugs; initially this was undertaken with CQ. Two i.v. doses of $2 \times 10^{4} P$. yoelii sporozoites $+\mathrm{CQ}$ induced sterile protection against homologous sporozoite challenge [45]. When a single dose of PQ was administered on the day of immunization, protection was not observed, indicating that the presence of liver-stage parasites is essential for inducing protective immunity. PQ treatment 
eliminated $>90 \%$ of liver-stage parasites. In this model, both $\mathrm{CD}^{+}$and $\mathrm{CD}^{+} \mathrm{T}$-cells contributed to the protection through induction of IFN- $\gamma$ and nitric oxide [45]. Protective immunity was directed at liver-stage parasites with limited protection against blood-stage parasite challenge [45].

The P. yoelii sporozoite IDC approach was also investigated with PQ alone [26]. BALB/c mice were injected with three doses of $P$. yoelii sporozoites. One dose of PQ was administered at the time of each sporozoite injection or at various time points up to 36 hours post-injection, and a second PQ dose was administered 48 hours after each sporozoite injection. After PQ treatment, $90 \%$ of the parasites were eliminated within 42 hours of infection. In contrast to the P. yoelii sporozoite $+\mathrm{CQ}$ immunization study, where PQ administration on the day of immunization prevented successful vaccination [45], PQ in this instance did not preclude induction of immunity as immunized mice were protected against homologous sporozoite challenge [26]; protection was maintained for at least 3 months. Immunity was stage-specific, with all immunized mice succumbing to a blood-stage challenge.

The potential direct contribution of CQ to the efficacy of the sporozoite + CQ regimen was examined for P. yoelii [46]. To investigate the possibility that CQ may be prolonging liverstage development which could result in enhancement of protective immune responses, liver parasite load was measured in CQ-treated and untreated mice infected with luciferaseexpressing P. yoelii sporozoites. This did not significantly differ between the two groups at any of the time points from 42-64 hours post immunization. Furthermore, parasites were detected in the blood of mice from both groups at the same time. While this study showed that CQ does not eliminate or delay the development of liver-stage parasites, it does not exclude CQ impacting on other aspects of liver-stage biology e.g. the repertoire of expressed antigens [46]. 
Sporozoite IDC with MQ was also evaluated with $P$. yoelii [47]. Two i.v. doses of $10^{4} P$. yoelii sporozoites (14 days apart) with concurrent MQ treatment (5-day regimen) induced antibodies against homologous sporozoites and $\mathrm{CD}^{+} \mathrm{T}$-cell-mediated sterile immunity following homologous sporozoite challenge. Following heterologous challenge with $P$. vinckei sporozoites, partial protection was observed with a 2 day delay in patency and a $92 \%$ reduction in peak parasitemia [47]. A significant reduction in liver parasite burden following both homologous and heterologous challenge was observed, although to a lesser degree with P. vinckei.

To investigate $P$. yoelii sporozoite IDC with other anti-malarial drugs, $10^{4} P$. yoelii sporozoites were administered to Swiss mice under curative doses of arteether (a semisynthetic derivative of artemisinin), MQ, Az or PQ [24]. Arteether and MQ target the parasite's blood-stage, Az targets both the liver-stage and blood-stage and PQ targets the liver-stage. Protection was not observed in mice even after five immunizations with P. yoelii sporozoites + PQ. Under arteether and MQ cover, delayed patency in blood-stage parasitemia following $10^{5}$ homologous sporozoite challenge was observed after the 4 th immunization and sterile protection against sporozoite challenge was achieved in mice that received five immunizations. Sterile protection against $10^{5}$ sporozoite challenge in mice immunized under Az cover was observed after four IDC. The protection induced in mice immunized with CPS under arteether, MQ or Az was stage-specific as the immunized mice developed blood-stage infection following homologous challenge with $10^{6} \mathrm{pRBCs}$.

The potential modulation of vaccine-induced pre-erythrocytic immunity by a subsequent blood-stage infection was also examined for P. yoelii sporozoite + MQ immunization [47]. To evaluate the effect of blood-stage malaria infection on pre-erythrocytic immunity, two weeks after the final immunization, mice were infected with homologous or heterologous $(P$. vinckei) blood-stage parasites. This subsequent parasite exposure to a homologous or 
heterologous blood-stage malaria infection did not abrogate protection to subsequent homologous sporozoite challenge in this rodent model.

\subsubsection{Immune correlates of protection and cross-stage immunity induced by $P$. yoelii} sporozoite infection and drug cure

Using antibody-mediated cell depletion, immunity induced by two i.v. doses of P. yoelii sporozoites $+\mathrm{CQ}$ in $\mathrm{BALB} / \mathrm{c}$ mice was shown to be dependent on $\mathrm{CD}^{+}$and $\mathrm{CD}^{+}{ }^{+}$-cells; IFN- $\gamma$ and NO were also critical effectors [45]. In a two dose $P$ yoelii sporozoite + MQ regimen, $\mathrm{CD}^{+} \mathrm{T}$-cells were also associated with protective immunity in BALB/c mice. In a further study, a single immunization with P. yoelii sporozoites + CQ (10 days of CQ) did not require $\mathrm{CD}^{+} \mathrm{T}$-cells for protection against homologous sporozoite challenge [48]. Following immunization, very low levels of blood-stage parasites persisted in the blood and this transient parasitemia after CQ treatment was associated with protection for this one-dose regimen; increasing CQ dosing to 25 days reduced the efficacy of the vaccine. Persistence of parasites also enabled the generation of antibody responses against blood-stage parasites. While antibody-mediated depletion of $\mathrm{CD} 4^{+} \mathrm{T}$-cells commencing at the time of immunization resulted in a fulminant blood-stage infection, depleting $\mathrm{CD}^{+}$or $\mathrm{CD}^{+} \mathrm{T}$-cells in immunized mice prior to challenge with $\mathrm{pRBC}$ did not impact protective cross-stage immunity. Thus, in this one immunization dose regimen, protection appeared to be independent of T-cells and relied instead on antibodies against the blood-stage parasite.

The induction of cross-stage immunity is an ideal goal [49]. Antigenic targets that are shared between liver- and blood-stage parasites are central to a malaria vaccine that provides crossstage protection. It is also possible that due to the nature of the IDC approach, exposure to low numbers of blood-stage parasites may induce cross-stage immunity. Although not 
observed in the $P$. berghei studies (Section 2.1.1.1), it was examined in the context of $P$. yoelii sporozoite IDC.

$\mathrm{BALB} / \mathrm{c}$ mice were immunized with three i.v. doses of $10^{4} P$. yoelii sporozoites and drug cure with either CQ or artesunate [22]. One month after vaccination, mice were challenged with homologous sporozoites or pRBCs. As expected, P. yoelii sporozoite + CQ immunization induced sterile protection against sporozoites. Following blood-stage parasite challenge, $62 \%$ of mice were sterilely protected whereas the remainder had a 1-4 day delay in the onset of parasitemia. P. yoelii sporozoite infection with artesunate induced total sterile protection against sporozoite challenge and delayed the onset of blood-stage infection in $73 \%$ of mice. Both groups had pre-erythrocytic stage-specific $\mathrm{CD} 8^{+}$effector memory T-cell responses and hepatic $\mathrm{CD}^{+}$T-cells that demonstrated cytotoxicity against liver stage parasites in vitro. Both groups also had antibodies that recognized pre-erythrocytic antigens, but only $P$. yoelii sporozoite + CQ immunization induced high levels of antibodies that recognized merozoite surface protein 1 (MSP1) and schizont lysate. The greater degree of cross-stage protection observed with the $P$. yoelii sporozoite + CQ immunization regimen may be explained by the differential effects of the anti-malarial drugs impacting on parasite persistence. Artesunate is a fast-acting drug and is active against all asexual blood-stages of the parasite, including early ring stages, whereas CQ acts mainly on trophozoite-stage parasites which could prolong exposure to low levels of blood-stage parasites. This is supported by the differential recognition of blood-stage antigens with the two sporozoite IDC regimens. The contribution of immune responses against shared antigens to this protection is unknown. Cross-stage immunity induced by the $P$. yoelii sporozoite + MQ regimen has also been observed, with significant protection observed against homologous and to a lesser degree, heterologous $(P$. vinckei) blood-stage challenge [47].

\subsubsection{Factors affecting induction of protective immunity: route of administration}


Unlike the $P$. berghei sporozoite IDC regimen (section 2.1.1.3), in the P. yoelii model, vaccine efficacy against liver-stage parasites was not dependent on the route of administration [50]. The liver parasite burdens following i.d. or i.v. challenge in mice immunized with two i.d. doses of $2 \times 10^{3}$ P. yoelii sporozoites + MQ were not significantly different from mice immunized with two i.v. immunizations of $P$. yoelii sporozoites $+\mathrm{MQ}$ with the same regimen.

\subsubsection{P. chabaudi sporozoite infection and drug cure}

The sporozoite IDC approach has been investigated with the $P$. chabaudi rodent model using MQ and CQ. P. chabaudi is an excellent model to study strain-specificity of vaccine-induced immune responses, using the different clones of P.c. chabaudi. Two i.v. doses of P.c. chabaudi $\mathrm{CB}$ sporozoites + MQ were shown to suppress blood-stage parasite growth in immunized mice following sporozoite or blood-stage challenge with homologous P.c. chabaudi $\mathrm{CB}$ or heterologous P.c. chabaudi AJ five weeks later [51]. The protective immunity was less effective in controlling heterologous parasite growth, particularly following blood-stage challenge. Conversely, P. c. chabaudi AJ immunization only induced partial homologous protection following sporozoite and blood-stage challenge. Understanding why some parasite strains (e.g. P. c. chabaudi CB) may induce a broader immunity, and the mechanisms responsible for this, are critical for the rational development of whole parasite vaccines.

Cross-stage and strain-specificity of protection were examined in a further study. Mice were immunized three times with the bites of P.c. chabaudi AS infected mosquitoes under CQ cover followed by a challenge 100 days after the final immunization [52]. In this model, immunization by mosquito bites did not generate pre-erythrocytic immunity. Induction of pre-erythrocytic immunity required immunization with a large number of sporozoites 
$(10,000)$, presumably to enable the development of a sufficient number of liver-stage parasites which may enhance the immune repertoire with sufficient exposure to a broad range of immunodominant and sub-dominant antigens [52]. P. chabaudi sporozoites administered by mosquito bites + CQ did however induce protection against homologous and heterologous (P. chabaudi $\mathrm{CB}$ ) blood-stage challenge, manifesting as reduced peak parasitemia and enhanced parasite clearance rates. Homologous immunity was again more effective than heterologous immunity. This study highlights a number of important variables that can impact on the specificity of the immune response and the efficacy of the vaccine including route of infection and antigen dose.

\subsubsection{P. knowlesi sporozoite infection and drug cure}

P. knowlesi is a zoonotic malaria parasite [53]. Investigating vaccine-induced immunity to a human malaria parasite in a non-human primate model enables interrogation of the immune response beyond the peripheral blood. Rhesus monkeys received three $P$. knowlesi sporozoite immunizations via mosquito bites $+\mathrm{CQ}$. Half of the immunized group (2/4) were completely protected against sporozoite challenge, whereas a delayed onset of parasitemia was observed in the remaining animals [54]. IgG specific for $P$. knowlesi whole sporozoites, circumsporozoite protein (CSP) and apical membrane antigen (AMA)-1 were detected in immunized monkeys, with higher sporozoite and CSP-specific antibodies observed in completely protected animals. Completely protected animals also had high frequencies of sporozoite-specific memory T-cells in blood, liver, spleen and bone marrow. Sporozoitespecific liver memory T-cells expressed the chemokine receptors CCR5 and CXCR6, whose ligands are involved in tissue homing, as well as the phenotypic marker of tissue-resident memory cells, CD69. The study design could not exclude that blood-stage immunity may have also contributed to the observed protection as the animals were exposed to transient blood-stage parasites during the immunization. 


\subsection{Clinical evaluation of $\boldsymbol{P}$. falciparum sporozoite infection and drug cure}

The nomenclature associated with the sporozoite IDC approaches was established following the first clinical study evaluating the protective efficacy of immunization with $P$. falciparum sporozoites administered by mosquito bites under CQ cover [55]. Sporozoite IDC studies using chemoprophylaxis with P. falciparum sporozoites administered by mosquito bite (CPS) or chemoprophylaxis vaccination with purified $P$. falciparum sporozoites administered by injection (CVac) have been widely conducted in malaria-naïve humans in a controlled clinical setting to evaluate safety, immunogenicity and efficacy [55-64]. Intravenous, i.d. and mosquito bites have all been used as routes of sporozoite administration. Intravenous and i.d. routes enable a precise dose as well as being practical and feasible for a large-scale vaccination program.

\subsubsection{Homologous protection induced by $P$. falciparum sporozoite infection and drug cure}

Malaria-naïve human volunteers were immunized three times with P. falciparum NF54 via 12-15 mosquito bites combined with CQ. Control subjects received bites from uninfected mosquitoes. Similar to the rodent models, a sub-microscopic parasitemia was detected in all ten vaccinees following the first immunization; blood-stage parasite burden decreased with each immunization. All CPS-CQ immunized subjects demonstrated complete sterile protection against homologous sporozoite challenge eight weeks after the final immunization [55]. No serious adverse events were recorded. Protection was shown to be long-lasting [57,59]. Four of six P. falciparum NF54 CPS-CQ immune volunteers were aparasitemic following re-challenge with homologous sporozoites 28 months after immunization, whereas the remaining two volunteers had delayed patency of parasitemia [59]. Potential immune correlates of protection are discussed in Section 2.2.4.

Similar to the rodent studies discussed previously, a further study was conducted to determine the stage-specificity of the protective immune response induced by P. falciparum CPS-CQ 
immunization [60]. Malaria-naïve individuals were immunized three times by mosquito bites with both $P$. falciparum NF54 and 3D7 (a clone of NF54) while on CQ prophylaxis; control subjects received CQ only. Volunteers were challenged i.v. with P. falciparum 3D7 pRBCs or by mosquito bites with $P$. falciparum $3 \mathrm{D} 7$ sporozoites. As the i.v. blood-stage challenge completely bypasses the liver stage, this allowed separate assessment of protective immune responses against each life-cycle stage. Complete sterile protection was observed in vaccinees who received the sporozoite challenge whereas those who received the blood-stage challenge developed parasitemia and had similar prepatent periods and blood-stage multiplication rates as the CQ-treated controls. Despite exposure to low levels of blood-stage parasite during the immunization phase immediately prior to challenge, antibodies against blood-stage antigens were detected in only one vaccinee and IgG from vaccinees did not display $P$. falciparum growth inhibitory activity in vitro. Following blood-stage challenge, IFN- $\gamma$ and the

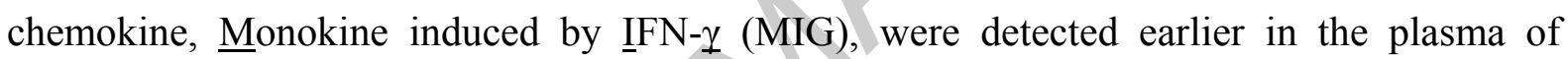
vaccinees compared with controls.

A further study also examined whether exposure to blood-stage parasites during CPS-CQ is required for vaccine efficacy against homologous challenge. In malaria-naïve individuals, three immunizations of CPS-CQ with 12-15 P. falciparum-infected mosquito bites at monthly intervals in malaria-naive individuals was directly compared with CPS-PQ/CQ, where a single dose of PQ was administered a day after sporozoite administration to kill the liverstages and completely prevent the development of blood-stage parasites [63]. Controls received non-infectious mosquito bites and PQ/CQ. During the immunization phase, only one individual in the CPS-PQ/CQ remained qPCR negative, indicating that the dosing regimen of PQ was sub-optimal in this study as it did not prevent development of blood-stage parasitemia. Following sporozoite challenge, all CPS-CQ vaccinees had delayed parasite patency, but did not develop sterile immunity, as has been reported in other studies $[55,60]$. 
They did however develop greater blood-stage immunity than the CPS-PQ/CQ group. Interestingly, thirty percent $(3 / 11)$ of the CPS-PQ/CQ vaccinees demonstrated sterile protection. The lack of sterile protection in the CPS-CQ group was attributed to a higher sporozoite inocula per mosquito at challenge than was expected; this increased number of sporozoites may have overwhelmed protective immune responses.

The protective efficacy of $P$. falciparum CPS has also been examined with MQ [61], which like CQ does not affect liver-stage development. Malaria-naïve volunteers were immunized with three doses of $P$. falciparum NF54 sporozoites by mosquito bites (four weeks apart) while receiving MQ and this was compared with CPS-CQ in the same study. The control group received MQ only. Seventy percent of volunteers immunized with $P$. falciparum CPSMQ were protected, which was not significantly different to the $60 \%$ protection observed in individuals who received CPS-CQ. Antibody and cellular immune responses were also comparable between groups.

\subsubsection{Heterologous protection induced by $P$. falciparum sporozoite infection and drug cure}

The ability of $P$. falciparum CPS to induce heterologous protective immunity in humans has also been examined. Sixteen volunteers previously immunized with three rounds of 5, 10 or 15 P. falciparum NF54-infected mosquito bites with CQ prophylaxis and challenged with $P$. falciparum NF54 (West-Africa) sporozoites were re-challenged with the geographically and genetically distinct $P$. falciparum clone NF135.C10 (Cambodia) sporozoites, 14 months after the last immunization [65]. Three volunteers previously not protected against $P$. falciparum NF54 sporozoite challenge were also not protected against challenge with $P$. falciparum NF135.C10. Amongst thirteen volunteers who were completely protected against the original P. falciparum NF54 challenge, sterile protection against heterologous strain NF135.C10 was 
observed in 2 volunteers (one each from the 10 and 15 mosquito bites immunized groups). Delayed patency was seen in the other 11 volunteers, indicating partial protection. There are several possible explanations for these results. Protective immune responses may have decreased as the heterologous challenge was performed 14 months rather than the usual 2-5 months after the last immunization. Additionally, the different sporozoite immunization doses (varied between volunteers from 5 to 15 mosquito bites) could have resulted in differential heterologous protection as higher doses might be needed for long-lasting protection, particularly against heterologous strains. Similar dose-dependent protection was observed in a dose-ranging study where complete homologous protection was obtained in 4 out of 5, 8 out of 9 and 5 out of 10 volunteers after CPS-CQ immunization with 45, 30 and $15 P$. falciparum-infected mosquito bites respectively [62].

A further study examined heterologous protection in malaria-naïve individuals in a doubleblind, placebo-controlled trial of P. falciparum NF54 CPS-CQ immunization followed by challenge with homologous or heterologous (P. falciparum NF135.C10 or NF166.C8 (Guinea)) sporozoites, eighteen weeks later [66]. While 5/5 individuals demonstrated sterile protection following homologous challenge, this was only observed in 2/10 (NF135.C10) and 1/9 (NF166.C8) individuals who received heterologous challenge. Six out of ten challenged with NF135.C10 had a prolonged prepatent period compared to controls. The inhibitory activity of plasma for blocking intra-hepatic development of heterologous strains in vitro was significantly lower than the homologous strain but this did not correlate directly with protection. There was significant diversity demonstrated in the target antigens for CPS-CQinduced antibodies in the heterologous strains compared with P. falciparum NF54. Parasitespecific T-cell responses (IFN- $\gamma$ and granzyme B) were detected in vitro in all groups.

In the $P$. falciparum CPS approach, modest protection against heterologous challenge has been observed $[65,66]$. Recent work has examined the biological heterogeneity of the three 
clinical isolates used in these studies to better understand this strain-specific vaccine-induced protection and the implications for vaccine efficacy in malaria endemic regions. Differences in invasion and replication within human hepatocytes in vitro were identified [67]. This differential infectivity was directly correlated with the magnitude of the first wave of bloodstage parasites in vivo and correlated inversely with the prepatent period. This in vitro and in vivo data support that a greater number of blood-stage parasites were released from the livers of individuals infected with NF135.C10 and NF166.C8 compared with NF54, which will impact on the prepatent period and must be considered when interpreting any data from studies using these isolates. Their genetic diversity was also examined, with tens of thousands of variants (including SNPs, indels and small structural variants) detected between the three isolates and this was reflected in variability in immunologically important regions of the genome which has the potential to impact on vaccine efficacy [68]. At a whole genome level, these isolates were shown to be a reliable proxy for the strains circulating in the geographic region from which they originated [68]. While it may be possible to further optimize the vaccine dose and schedule to improve vaccine efficacy against heterologous challenge [56], the development of a geographically-targeted multi-strain vaccine may also be required for high levels of protective efficacy in the field.

\subsubsection{Factors affecting induction of protective immunity following P. falciparum sporozoite} infection and drug cure: route of administration, immunization dose and frequency

As rodent studies demonstrated that the route of administration of sporozoites can impact on the generation of protective immunity (sections 2.1.1.3 and 2.1.2.3), this was also investigated for $P$. falciparum. The i.d. administration of cryopreserved, infectious $P$. falciparum sporozoites (Sanaria ${ }^{\circledR}$ PfSPZ Challenge) was tested for its safety and immunogenicity in the context of CVac with CQ [64]. Volunteers were immunized i.d. with three or four doses of $7.5 \times 10^{4}$ sporozoites while taking CQ; controls received saline. Prior 
to challenge, vaccinees had weak antibody responses against CSP, liver stage antigen 1 (LSA1), MSP1 and no detectable cellular immune responses against $P$. falciparum $\mathrm{pRBC}$ or sporozoites. Following challenge by $P$. falciparum NF54-infected mosquito bites, 2/8 vaccinees did not develop microscopically patent parasitemias however, they both developed transient parasitemias detectable by qPCR. The lack of protection was attributed to suboptimal parasite exposure during the i.d. immunizations with insufficient numbers of sporozoites migrating to and developing in the liver. A further CVac study evaluated i.v. administration of sporozoites (Sanaria ${ }^{\circledR}$ PfSPZ challenge) to malaria-naïve volunteers while taking CQ [56]. It revealed that the protective efficacy was not only dependent on route of administration but also on the immunization dose and dosing frequency. A CVac regimen of three i.v. doses of $3.2 \times 10^{3}, 1.28 \times 10^{4}$ or $5.12 \times 10^{4} P$. falciparum sporozoites + CQ at 28 day intervals induced sterile protection against i.v. challenge with homologous sporozoites in $33 \%, 67 \%$ and $100 \%$ of volunteers, respectively. When the completely protective regimen was administered at 5- and 14-day intervals instead of 28-day intervals, protection against $2 \times 10^{3}$ PfSPZ i.v. challenge was achieved in only $63 \%$ and $67 \%$ of volunteers. Increasing the number of sporozoites in the vaccine may improve protection in these accelerated vaccination schedules. Therefore, route of administration, antigen dose as well as timing of immunizations are major determinants of the protective efficacy of $P$. falciparum sporozoite IDC approaches.

2.2.4 Immune correlates of protection induced by $P$. falciparum sporozoite infection and drug cure

\subsubsection{Cellular correlates of immunity}

Numerous studies have characterized the immune response induced by $P$. falciparum sporozoite IDC approaches; this may enable identification of immune correlates of 
protection. Protective immunity against homologous sporozoite challenge is associated with the induction of pluripotent effector memory T-cells [55]. In protected vaccinees, a significant increase in the proportion of multiple cytokine (IFN- $\gamma$, TFN- $\alpha$, IL-2)-producing pluripotent effector memory T-cells in response to pRBCs was observed at the pre-challenge timepoint. In a sub-set of individuals who were challenged 28 months later, long-term persistence of parasite-specific pluripotent effector memory T-cell responses was detected prior to re-challenge [59], although the parasite-specific cytokine responses in the $2 / 6$ individuals with delayed onset of parasitemia following challenge were not significantly different from the $4 / 6$ who were completely protected. In a further study, protection was also associated with increased expression of markers of cellular cytotoxicity [62]. Higher proportions of $\mathrm{CD}^{+}$T-cells expressing the degranulation marker CD107a and granzyme Bproducing $\mathrm{CD}^{+}$T-cells were observed at the pre-challenge time point following in vitro restimulation with P. falciparum pRBC.

The comparative $P$. falciparum CPS-CQ and CPS-MQ immunization study, which induced complete protection following homologous challenge in $60 \%$ and $70 \%$ of vaccinees respectively [61], also suggested a potential role for $\gamma \delta$ T-cells, Th1 and cytotoxic responses in vaccine-induced protection [69]. A possible suppressive role of regulatory T-cells was also identified, as proliferation of this cell population was significantly higher after the second immunization in subjects not protected against sporozoite challenge.

Most studies have identified immune responses associated with protection by comparing prechallenge samples from fully protected vaccinees with the control group or comparing immune responses in samples from vaccinees collected at baseline and pre-challenge. This approach primarily identifies immune responses induced by vaccination; they may or may not be mediating protection. In a recent study, cellular immune responses were compared between protected and unprotected individuals vaccinated with P. falciparum CVac with CQ 
[56]. Pre-challenge, $\mathrm{CD}^{+}{ }^{+}$T-cells that simultaneously produced IFN- $\gamma$, IL-2 and TNF- $\alpha$ were more prevalent in subjects who did not develop parasitemia, whereas $\mathrm{CD}^{+} \mathrm{T}$-cells that produced TNF- $\alpha$ only were more frequent in those who became parasitemic. Sterile protection was strongly associated with a higher frequency of $\mathrm{pRBC}$-specific polyfunctional IFN- $\gamma$, IL-2 and TNF- $\alpha$ producing $\mathrm{CD}^{+}{ }^{+}$T-cells. Protection was weakly associated with pRBC-specific IFN- $\gamma$ producing $\mathrm{CD} 8^{+} \mathrm{T}$-cells.

Whole blood transcriptome profiling by RNA-seq provides a broad, unbiased approach for evaluating the dynamics and composition of immune responses [70]. In individuals completely protected against homologous sporozoite challenge, differential expression of genes related to T-cell, NK-cell, protein synthesis and mitochondrial processes were detected four weeks after the first CPS-CQ immunization, suggesting induction of cytotoxic cellular responses. These signatures were not detected in protected subjects four weeks after the second immunization or 132 days after the third immunization. Similar signatures were however detected in these individuals three weeks following sporozoite challenge, suggesting an ongoing T-cell memory response after sporozoite exposure and in the absence of detectable parasitemia. Interestingly, evidence of immune interactions between different components of the immune system were observed in protected individuals in the weeks following the second and third immunizations. In contrast, in non-protected subjects, transcriptomic changes were detected straight after the third immunization and on their day of drug treatment (in the presence of patent parasitemia) with upregulation of interferon and innate inflammatory genes and downregulation of B-cell signatures. These data provide $P$. falciparum CPS-CQ-induced molecular signatures associated with protection that can be interrogated in greater detail in future studies.

\subsubsection{Humoral correlates of immunity}


The induced antibody response, including breadth, magnitude and functionality, has been examined in different studies to determine the role of antibodies in P. falciparum sporozoite IDC approaches and to identify potential antibody correlates of protective immunity.

Antibodies may play a role in protection, but their mechanism of action in pre-erythrocytic immunity may extend beyond direct neutralisation of sporozoites or inhibiting invasion of sporozoites. An additional mechanism of action is activation of complement. The complement system plays a role in phagocyte recruitment, pathogen opsonization and pathogen lysis via downstream C3 complement protein deposition. Antibody-mediated complement activation has been identified as an important mechanism for inhibiting invasion of $P$. falciparum merozoites, which facilitates a reduction in $P$. falciparum blood-stage replication and prevention of clinical disease [71]. To investigate this, antibodies in sera from P. falciparum NF54 CPS-CQ immunized volunteers, who were completely protected from homologous sporozoite challenge, were evaluated for their capacity to activate complement [72]. CPS-CQ immunization induced sporozoite-specific complement-fixing IgM and IgG1 antibodies that were capable of activating the classical complement pathway resulting in functional lysis of sporozoites and a reduction in homologous (NF54) and heterologous (NF135.C10) sporozoite infectivity of human hepatocytes in vitro. These data highlight the role of complement as an immune effector mechanism in pre-erythrocytic immunity induced by $P$. falciparum CPS-CQ.

Using a proteome microarray containing 809 antigens, the reactivity of pre-challenge plasma from malaria-naïve individuals who were completely protected following $P$. falciparum CPSCQ immunization was compared with semi-immune individuals from a malaria endemic area [73]. There was substantial variation in the number of antigens recognized in the $P$. falciparum CPS-CQ-immunized individuals, however recognition of the pre-erythrocytic antigens CSP and LSA1, were common in all protected volunteers. Strikingly, while there 
were 60 antigens that overlapped between the two groups, the serological recognition profile of CPS-CQ immunized volunteers was skewed towards pre-erythrocytic antigens while plasma from naturally exposed semi-immune Kenyan individuals was biased towards recognition of asexual blood-stage antigens. These results shed light on the antibody profile induced in immunized volunteers and the identification of antigens for further investigation as potential targets of protective immunity.

A further study also screened pre-challenge sera from malaria-naïve individuals who developed long-lasting sterile immunity following three immunizations of $P$. falciparum CPS-CQ for antibody functionality and against a broad repertoire of $P$. falciparum antigens that were expressed during the pre-erythrocytic stage [74]. Pooled sera from protected volunteers inhibited liver-stage development in vitro whereas there was limited invasion inhibitory activity specific for blood-stage parasites (2/9 vaccinees). Overall, there was a broad and varied Plasmodium antigen-specific antibody response detected in protected individuals. Three antigens: Pf merozoite apical erythrocyte binding ligand (MAEBL) (sporozoite, liver, asexual blood-stage expression), Pf thrombospondin-related adhesive protein (TRAP) (sporozoite, liver, asexual blood-stage expression) and Pf Schizont Egress Antigen-1 (SEA1) (sporozoite, liver-stage expression), were recognized by antibodies present in the sera of $6 / 9,8 / 9,9 / 9$ protected individuals. These antibodies were not present or were variably present at low levels in mock-immunized individuals. Antibodies raised against PfMAEBL demonstrated invasion inhibitory activity against sporozoites in vitro. These data suggest a potential role for these antibodies in protection.

In a recent study, it was possible to compare antibody responses in protected and unprotected individuals vaccinated with $P$. falciparum CVac with CQ [56]. Antibody responses were evaluated using a $P$. falciparum proteome array expressing 7,455 malaria peptides, standard protein ELISAs, Immunofluorescence Assays on whole sporozoites and assessment of 
invasion inhibitory activity for sporozoites. There was no significant association of any antibody responses with protection. The authors hypothesized that antibodies may play a role in protection but are not the primary effector. This same proteome array was also used to screen a larger number of sera from protected and unprotected vaccinees enrolled in multiple studies [75]. Antibody responses against six antigens were associated with susceptibility and responses against six entirely novel antigens were associated with protection. Together, this multi-antigen combination predicted protection in the CPS-immunized individuals with $83 \%$ sensitivity and $88 \%$ specificity. High reactivity against the immunodominant antigens CSP, LSA1, MAEBL, TRAP, SEA1 was not associated with protection individually or in combination; suggesting that they may be markers of exposure rather than protection for the P. falciparum CPS-CQ regimen [75]. This is particularly interesting for CSP, which is the major parasite antigen contained within the most advanced malaria vaccine, Mosquirix ${ }^{\mathrm{TM}}$ (RTS,S/AS01) [76].

\subsection{Plasmodium infection and drug cure with asexual blood-stage parasites}

Infection and Drug Cure with asexual blood-stage parasites (referred to as controlled infection immunization (CII) in this review) has been explored to a lesser extent compared with sporozoite IDC. Asexual blood-stage parasite CII is administered via i.v. injection of pRBCs and is typically followed by anti-malarial drug administration. Protection against blood-stage challenge is determined by the development of blood-stage parasitemia, bloodstage parasite levels, parasite multiplication rates and/or clearance rate of parasites.

\subsection{Pre-clinical evaluation of $P$. chabaudi, $P$. yoelii and $P$. vinckei asexual blood-stage parasite infection and drug cure}

\subsubsection{Homologous and heterologous protection}


Blood-stage CII has been investigated using different rodent malaria parasites in combination with various anti-malarial drugs such as $\mathrm{MQ}$, atovaquone-proguanil (Malarone), CQ, doxycycline and Az.

To examine strain-specific protective immunity induced by blood-stage CII, $P$. chabaudi CII was examined using a combination of $10^{5}$ P. c. chabaudi AS CII with Malarone initiated 48 hours after parasite inoculation, which prevented the development of a microscopically detectable blood-stage infection. Three i.v. immunizations of the P. c. chabaudi AS CII protected mice against both homologous and heterologous (P. c. chabaudi CB) blood-stage parasite challenge [77]. In all vaccinated mice following challenge, low level parasitemias were detected that were significantly lower than in the naïve control mice. Peak parasitemias in mice challenged with heterologous parasites were significantly higher than in mice challenged with homologous parasites. Serum from CII mice contained IgG specific for AS and $\mathrm{CB}$ pRBCs and conserved merozoite surface antigens, as measured by ELISA and immunofluorescence assays, but lacked IgG specific for variant surface antigens (VSA) of AS pRBCs. This is in direct contrast to serum from mice who experienced patent, selfresolving blood-stage malaria infections which also contained significant levels of $\operatorname{IgG}$ specific for the VSA. These data suggest that the VSA are less immunogenic or are expressed at relatively lower levels than the other malaria antigens. It may be that by reducing malaria parasite antigen concentrations in the blood-stage CII mice compared to those who experienced a patent blood-stage infection, this negatively impacts on the generation of adequate $\mathrm{T}$ help for generation of effective VSA-specific antibody responses [77]. Mice immunized by this blood-stage CII were able to control the parasitemia following challenge, suggesting that variant-specific antibodies were not critical for protection. Prominent Th1 cell-mediated immune responses (IFN- $\gamma^{+} \mathrm{CD} 4^{+}$lymphocytes) were detected in immunized mice. Splenocyte proliferative responses to $P$. c. chabaudi $\mathrm{AS}$ and $\mathrm{CB} \mathrm{pRBC}$ were similar in 
immunized mice, suggesting that the blood-stage CII-induced cell-mediated immunity was targeting conserved antigens.

Strain-transcending immunity was also demonstrated with P.c. chabaudi clones used in combination with $\mathrm{MQ}$, with treatment initiated 5 days after the injection of blood-stage parasites [78]. Mice received one or two doses of blood-stage CII with P.c. chabaudi AS or $\mathrm{CB}$ and following the last immunization they received a mixed strain blood-stage parasite challenge with equal numbers of the homologous and heterologous parasite strains. In mice that received a single dose of CII, there was a partial reduction in parasitemia compared with naïve mice, with the blood-stage parasitemias of each parasite strain reduced to equal degrees. In mice that received two doses of CII, the reduction in parasitemias was greater and homologous strain parasitemia reduced faster than the heterologous strain for all combinations of P. c. chabaudi clones tested (AS, CB, AJ, AQ). This suggests that the strainspecific component of immunity induced by blood-stage CII may depend on immune memory.

Cross-species immunity has been demonstrated in the context of $P$. yoelii blood-stage CII with MQ. Mice immunized with one dose of $10^{6}$ P. yoelii blood-stage CII with MQ (starting on day 5 post-inoculation) were completely protected against homologous blood-stage infection; and the peak parasitemias in mice challenged with a heterologous species $(P$. vinckei lentum) were significantly reduced compared to parasitemias in MQ-treated control mice [47]. Similar results were obtained when mice were immunized with $10^{6} \mathrm{P}$. vinckei blood-stage CII with MQ and challenged with homologous or heterologous parasites. Immunization induced significant levels of antibodies specific for homologous species crude parasite antigen and much lower levels against heterologous species crude parasite antigen. 
A further study determined that the timepoint for initiation of anti-malarial drug treatment impacted on the development of cross-species protective immunity in the context of bloodstage CII with PYR [79]. Mice inoculated with $5 \times 10^{4}$ pRBCs of the non-lethal strain $P$. chabaudi AS and drug-treated on day-5 failed to develop species-transcending protection against the more virulent $P$. yoelii 17XL, with all mice succumbing to infection. In contrast, when drug treatment was administered on day-7 post-parasite inoculation, mice survived $P$. yoelii challenge with lower parasitemia levels. A predominant Th2-type response with higher IL-4 and IL-10 levels and lower IFN- $\gamma$ expression was associated with protection against lethal infection.

For blood-stage CII to be a viable vaccine approach it is critical however, that anti-malarial drug treatment is initiated at the same time that the parasite is inoculated. A delay in treatment initiation and relying on individuals to return for drug treatment is a safety concern. For this reason, blood-stage CII was examined with the delayed death anti-malarial drugs doxycycline and Az [80]. Delayed death drugs target the apicoplast of the parasite, inhibiting the growth of progeny of the parasites thereby allowing the parasites to persist for an extra replication cycle [81]. Initially, BALB/c and C57BL/6 mice received three immunizations with $10^{6}$ or $10^{7} P$. chabaudi AS or P. yoelii YM blood-stage parasites, with doxycycline treatment initiated on the same day $(50 \mathrm{mg} / \mathrm{kg}$ for 7 days) [80]. Strong protective immunity was observed against homologous challenge. Heterologous protection was also observed in P. chabaudi AS-immunized BALB/c and C57BL/6 mice and P. yoelii YM-immunized C57BL/6 mice, although this was not as strong as homologous protection. Depletion studies demonstrated that $\mathrm{CD}^{+}$T-cells played a critical role in homologous protection in the $P$. chabaudi AS model with a broad Th1 cytokine response (IFN- $\gamma$, TNF- $\alpha$, IL-2) correlated with the protection. While in the $P$. yoelii model, antibodies significantly contributed to protection. The persistence of parasites, enabled by the use of doxycycline, was crucial for 
the induction of protective immunity. Mice receiving three P. chabaudi or P. yoelii CIIs with doxycycline had detectable parasite DNA for at least one week following immunization with complete clearance by day 14 . In comparison, mice receiving three P. chabaudi CIIs with Malarone or P. yoelii CII with PYR, exhibited an immediate decline in parasite levels and parasites were undetectable by day 2 as measured by qPCR. Following homologous challenge, mice that were immunized with CII and doxycycline demonstrated superior protection to mice that received CII with the faster-acting drugs.

As a vaccine approach, to ensure patient safety, it would be desirable to administer the antimalarial drug not only at the time of parasite inoculation, but also in a single dose. For this purpose, P. yoelii CII was next examined with the delayed death drug Az which was administered in a single dose at the same time as parasite inoculation [80]. Mice receiving a single $P$. yoelii CII with Az $(500 \mathrm{mg} / \mathrm{kg})$ were strongly protected from a homologous bloodstage challenge one month later. The need for repeated infection and treatment cycles brings a new challenge for the implementation of CII in a clinical setting as well as in malariaendemic areas. Single CII with a single dose of the drug, as reported in this study, is ideal for a blood-stage CII vaccine.

\subsubsection{Cross-stage immunity}

Induction of cross-stage immunity by blood-stage CII has been examined in a limited number of studies. BALB/c mice immunized with a single dose of $1 \times 10^{6}$ P. yoelii or P. vinckei lentum blood-stage parasites in combination with MQ (commenced on Day 4 or 5 following parasite inoculation) were challenged i.v. with $P$. yoelii sporozoites [47]. The liver-stage parasite burdens in immunized mice at 42 hours post-challenge were not significantly different from naïve, challenged mice indicating a lack of cross-stage immunity. 
In contrast, immunization with one dose of $10^{6}$ or two doses of $10^{5} P$. yoelii blood-stage CII with daily CQ treatment for 10 days starting on the day of pRBC administration was shown to induce protection against sporozoite challenge; a single dose of $10^{6}$ also completely protected against blood-stage challenge [82]. Protection against sporozoite challenge was mediated by T-cells that were inhibitory to liver-stage parasites and antibodies targeting any blood-stage parasites that emerged from the liver. The effect of $\mathrm{CD}^{+}$and $\mathrm{CD} 8^{+} \mathrm{T}$-cells was partly mediated by IFN- $\gamma$ and was completely dependent on nitric oxide.

\subsection{Clinical studies with $P$. falciparum blood-stage parasite infection and drug cure}

There has been limited investigation of $P$. falciparum blood-stage CII. Malaria-naïve individuals received four cycles of 30 viable $P$. falciparum $3 \mathrm{D} 7 \mathrm{pRBC}$ at five-weekly intervals, with three infections treated with Malarone prior to patency (starting on day 8 postparasite inoculation) and the last infection treated with CQ on day 14 post-parasite inoculation [83]. This P. falciparum blood-stage CII induced protection as characterized by the absence of parasites or parasite DNA in the blood by qPCR in three of the four volunteers following administration of the final infection and diminished parasite growth in the fourth volunteer. It is possible that residual low levels of atovaquone detected in the volunteer's plasma may have contributed towards the protection [84]. The observed protection was associated with proliferative $\mathrm{CD} 4^{+}$and $\mathrm{CD} 8^{+} \mathrm{T}$-cells responses against $\mathrm{pRBC}$ in vitro, along with the IFN- $\gamma$ production and higher induction of nitric oxide synthase in peripheral blood mononuclear cells. Immunity was independent of humoral responses as parasite-specific antibodies to either whole parasite or recombinant $P$. falciparum proteins (MSP1-19, MSP2, AMA1) were not detected.

A further study examined parasite persistence and immunogenicity in blood-stage CII with doxycycline. A single immunization with $3 \times 10^{6}$ P. falciparum-infected RBCs followed by 
doxycycline treatment (100 mg/day for 21 days) commencing $1 \mathrm{hr}$ after parasite administration increased parasite-specific cellular responses $\left(\mathrm{CD}^{+}, \mathrm{CD}^{+}\right.$, and $\gamma \delta \mathrm{T}$-cells, along with IFN- $\gamma$ and TNF) [80]. Three of four individuals also produced parasite-specific IgM antibodies. This CII approach was well tolerated. Three of the four volunteers developed parasitemia detectable by qPCR; parasites were only detectable for 2 growth cycles in one of these. Persistent low levels of parasites, which were controlled by doxycycline treatment, were observed in the remaining two volunteers. However, after completion of doxycycline, the parasitemia in these volunteers increased and they required rescue treatment on day 28 with artemether-lumefantrine as per the requirements of the study protocol. Further studies are required to determine if this CII protocol induces protective immunity in humans.

\subsection{Concluding remarks}

The development of a highly effective malaria vaccine is essential for the control and eventual eradication of the malaria parasite. While the low and variable protection observed for sub-unit vaccine candidates in the field has been disappointing, IDC-based whole parasite vaccines are a feasible alternative strategy for the development of a malaria vaccine. Much progress has been made with the sporozoite IDC approaches, with strong sterile protection demonstrated against challenge with homologous parasites in malaria-naïve individuals. Route of administration, sporozoite dose, choice of anti-malarial drug and timing of drug administration were all critical variables for the induction of protective immunity (Table 1). There was limited evidence of cross-stage immunity. Further optimization, including the assessment of higher parasite doses, will be required to enhance strain-transcending immunity to ensure it is able to offer a broad protection. While less progress has been made with the asexual blood-stage CII vaccine approach and there has been limited clinical evaluation, the existing pre-clinical and clinical data supports the induction of strong homologous protective immunity (Table 1). Persistence of the blood-stage parasite following blood-stage CII is 
critical for the induction of strong protective immunity. Furthermore, recent data support an optimized blood-stage CII regimen with concurrent administration of the parasite and a single-dose anti-malarial drug, which addresses a major safety concern with this vaccine strategy [80]. Additional clinical studies will be needed to evaluate the protective efficacy of the blood-stage CII vaccine against homologous and heterologous parasites. While further development is required for an IDC vaccine, it remains a promising approach for the development of a broadly protective malaria vaccine.

\subsection{Expert Opinion}

Development of a highly effective malaria vaccine that is capable of inducing a broad protective immunity against the many different parasite strains circulating in malaria endemic areas would play a key role in the eradication of the malaria parasite. Plasmodium IDC is one of a number of whole parasite malaria vaccine approaches that are currently being developed and at least in terms of the whole sporozoite approaches, it offers superior protection. In theory, inclusion of the whole parasite into the vaccine should limit the impact of antigenic diversity and immunological non-responsiveness; these factors contribute to the low efficacy that has been observed when sub-unit vaccine candidates have been tested in the field. To date, sporozoite IDC studies in rodents and malaria-naïve humans have demonstrated that while this approach can induce strong protection against homologous challenge, equivalent levels of heterologous immunity are not induced (Table 1). This may be addressed by further vaccine optimization. If heterologous immunity is limited by the dose of conserved antigenic target/s of protective immunity, then increasing the parasite dose may increase the straintranscending immune response. Alternatively, if the parasite's genetic diversity is also an impediment with this vaccine approach, then including multiple representative strains in the vaccine may be required. 
The data presented and discussed in this review reflect that clinical evaluation of IDC approaches for the development of a malaria vaccine have to-date focused on $P$, falciparum. The necessary reagents to develop both pre-erythrocytic [85] and blood-stage $P$. vivax [86] whole parasite vaccines are available, although there are additional considerations for $P$. vivax. These include: the challenges of long-term culture of $P$. vivax to generate sufficient material for a whole parasite blood-stage vaccine and the possibility of hypnozoite formation and infection relapse following immunization with sporozoites. While PQ or tafenoquine can be administered to clear these latent liver stages, there is a risk of PQ-induced hemolysis in G-6-PD deficient individuals. Multiple relapses in two participants in a $P$. vivax mosquito challenge study also highlighted human cytochrome P-450 isoenzyme 2D6 (CYP2D6) polymorphisms, which impact the efficacy of PQ by affecting the conversion of PQ to its active metabolite [87]. These are issues that will need to be addressed to enable the development of $P$. vivax-specific IDC-based vaccine approaches.

Substantial immunological interrogation aiming to identify mechanisms and correlates of immunity has been undertaken in a number of studies; results were not always consistent between studies (Table 1). Identifying an immune response/s that is reproducibly correlated with protection and understanding the mechanisms of immunity will be a major advantage in evaluating vaccine efficacy and informing any further vaccine optimization.

The IDC regimen employed for vaccination must also be appropriate for large-scale administration in malaria endemic areas. While sporozoite immunization by mosquito bites has been used for many studies so far, the use of purified sporozoites administered via an i.v. route will enable transitioning a sporozoite IDC-based vaccine approach into the field. The need for multi-dose administration of anti-malarial drugs is also a potential safety issue for this vaccine approach as it relies on self-administration or individuals returning to a health clinic for these drugs. Ideally, an anti-malarial drug that can be administered concurrently 
with the parasite in a single dose is required. Proof-of-concept studies in rodents, demonstrated that IDC with a single dose of drug (PPQ for sporozoite CII and Az for bloodstage CII) $[25,80]$ induced strong protective immunity. Alternatively, novel drug formulations that can reduce drug dosing frequency by modifying the rate of drug absorption may be developed to enable single dose administration. The lack of adherence to a multi-dose vaccination regimen is always a concern however, further optimization of the vaccination regimens may enable the number of vaccine doses to be reduced, particularly if natural boosting of vaccine-induced immune responses is observed.

Ultimately an IDC-based vaccine approach needs to be evaluated in malaria endemic areas in individuals who have been exposed to the malaria parasite. Few studies have examined the duration of protective immunity induced by an IDC-based approach in humans; for sporozoite CPS-CQ, protection against homologous challenge lasted for at least two years in malaria-naïve adults [59]. It will be essential to define this in malaria endemic areas in different age groups following exposure to diverse parasite strains and to determine whether this exposure is able to boost and maintain protective immune responses. This will be of particular interest as there is no evidence that the CSP-specific antibodies induced by the leading malaria vaccine, RTS,S/AS01, are boosted following natural exposure [88]. It is also unknown how the vaccine will perform in the presence of pre-existing Plasmodium-specific immune responses. Recent data from a Tanzanian study evaluating another whole parasite vaccine, the RAS vaccine (PfSPZ), in malaria-exposed adults, showed that despite substantial person-to-person variation in seroreactivity to an array of $P$. falciparum proteins at baseline, this reactivity profile remained largely unchanged following repeated PfSPZ vaccination [89]. This suggests that at least for this particular vaccine, only pre-existing antibody responses were boosted, and this was attributed to natural imprinting of the individual immune responses. It is unknown whether this will be relevant for cellular immune 
responses. The impact of this on the vaccine's protective efficacy is unclear, however, a Malian study observed that this PfSPZ vaccine had a protective efficacy of $48 \%$ by time to first positive blood smear and $29 \%$ by proportion of participants with at least one positive blood smear during a full transmission season [90]. This demonstrates that a whole sporozoite vaccine can induce protective immunity against naturally transmitted malaria infection in the presence of pre-existing Plasmodium-specific immune responses. The impact of pre-exposure will be a consideration for both the evaluation of whole parasite vaccines in malaria-exposed populations and its eventual application, as it may result in greater heterogeneity in the immune response and protective efficacy than observed in malaria-naïve individuals.

\section{References}

1. World Health Organization World malaria report 2019.

2. Tuteja R. Malaria - an overview. FEBS J. 2007 Sep;274(18):4670-9. 
3. Riveron JM, Tchouakui M, Mugenzi L, et al. Insecticide resistance in malaria vectors: an update at a global scale. In: Manguin S, Dev V, editors. Towards malaria elimination-a leap forward. London, UK: IntechOpen,; 2018. p. 149-173.

4. Conrad MD, Rosenthal PJ. Antimalarial drug resistance in Africa: the calm before the storm? Lancet Infect Dis. 2019 Oct;19(10):e338-e351.

5. Sagara I, Dicko A, Ellis RD, et al. A randomized controlled phase 2 trial of the blood stage AMA1-C1/Alhydrogel malaria vaccine in children in Mali. Vaccine. 2009 May $18 ; 27(23): 3090-8$.

6. Ogutu BR, Apollo OJ, McKinney D, et al. Blood stage malaria vaccine eliciting high antigen-specific antibody concentrations confers no protection to young children in Western Kenya. PLoS One. 2009;4(3):e4708.

7. Sirima SB, Cousens S, Druilhe P. Protection against malaria by MSP3 candidate vaccine. N Engl J Med. 2011 Sep 15;365(11):1062-4.

8. White MT, Verity R, Griffin JT, et al. Immunogenicity of the RTS,S/AS01 malaria vaccine and implications for duration of vaccine efficacy: secondary analysis of data from a phase 3 randomised controlled trial. Lancet Infect Dis. 2015 Dec;15(12):14508.

9. Olotu A, Fegan G, Wambua J, et al. Seven-Year Efficacy of RTS,S/AS01 Malaria Vaccine among Young African Children. N Engl J Med. 2016 Jun 30;374(26):251929.

10. Stanisic DI, Good MF. Whole organism blood stage vaccines against malaria. Vaccine. 2015 Dec 22;33(52):7469-75.

11. Stanisic DI, McCarthy JS, Good MF. Controlled Human Malaria Infection: Applications, Advances, and Challenges. Infect Immun. 2018 Jan;86(1):1-17.

12. Whitrow M. Wagner-Jauregg and fever therapy. Med Hist. 1990 Jul;34(3):294-310. 
13. Gartlehner G, Stepper K. Julius Wagner-Jauregg: pyrotherapy, simultanmethode, and 'racial hygiene'. J R Soc Med. 2012 Aug;105(8):357-9.

14. Collins W, Jeffery G. A retrospective examination of secondary sporozoite-and trophozoite-induced infections with Plasmodium falciparum: development of parasitologic and clinical immunity following secondary infection. Am J Trop Med Hyg. 1999;61(1_Supplement):20-35.

* Retrospective analyses of historical malariotherapy clinical studies demonstrating induction of partial clinical and/or anti-parasite immunity following deliberate malaria infection.

15. Doolan DL, Dobano C, Baird JK. Acquired immunity to malaria. Clin Microbiol Rev. 2009 Jan;22(1):13-36.

16. Trager W, Jensen JB. Human malaria parasites in continuous culture. Science. 1976 Aug 20;193(4254):673-5.

17. Chulay JD, Schneider I, Cosgriff TM, et al. Malaria transmitted to humans by mosquitoes infected from cultured Plasmodium falciparum. Am J Trop Med Hyg. 1986 Jan;35(1):66-8.

18. Billingsley P, Sim B, Bijker E, et al. Controlled human malaria infections using aseptic, purified cryopreserved Plasmodium falciparum sporozoites administered by needle and syringe. Malaria journal. 2015;13(1):P12.

19. Derbyshire ER, Mota MM, Clardy J. The next opportunity in anti-malaria drug discovery: the liver stage. PLoS Pathog. 2011 Sep;7(9):e1002178.

20. Bijker EM, Nganou-Makamdop K, van Gemert GJ, et al. Studying the effect of chloroquine on sporozoite-induced protection and immune responses in Plasmodium berghei malaria. Malar J. 2015 Mar 26;14(1):130. 
21. Friesen J, Matuschewski K. Comparative efficacy of pre-erythrocytic whole organism vaccine strategies against the malaria parasite. Vaccine. 2011 Sep 16;29(40):7002-8.

22. Peng X, Keitany GJ, Vignali M, et al. Artesunate versus chloroquine infectiontreatment-vaccination defines stage-specific immune responses associated with prolonged sterile protection against both pre-erythrocytic and erythrocytic Plasmodium yoelii infection. J Immunol. 2014 Aug 1;193(3):1268-77.

23. Lewis MD, Behrends J, Sa ECC, et al. Chemical attenuation of Plasmodium in the liver modulates severe malaria disease progression. J Immunol. 2015 May 15;194(10):4860-70.

24. Bhardwaj J, Siddiqui AJ, Goyal M, et al. Repetitive live sporozoites inoculation under arteether chemoprophylaxis confers protection against subsequent sporozoite challenge in rodent malaria model. Acta Trop. 2016 Jun;158:130-138.

25. Pfeil J, Sepp KJ, Heiss K, et al. Protection against malaria by immunization with nonattenuated sporozoites under single-dose piperaquine-tetraphosphate chemoprophylaxis. Vaccine. 2014 Oct 14;32(45):6005-11.

26. Putrianti ED, Silvie O, Kordes M, et al. Vaccine-like immunity against malaria by repeated causal-prophylactic treatment of liver-stage Plasmodium parasites. J Infect Dis. 2009 Mar 15;199(6):899-903.

27. Friesen J, Borrmann S, Matuschewski K. Induction of antimalaria immunity by pyrimethamine prophylaxis during exposure to sporozoites is curtailed by parasite resistance. Antimicrob Agents Chemother. 2011 Jun;55(6):2760-7.

28. Recht J, Ashley EA, White NJ. Use of primaquine and glucose-6-phosphate dehydrogenase deficiency testing: Divergent policies and practices in malaria endemic countries. PLoS Negl Trop Dis. 2018 Apr;12(4):e0006230. 
29. Bejon P, Andrews L, Andersen RF, et al. Calculation of liver-to-blood inocula, parasite growth rates, and preerythrocytic vaccine efficacy, from serial quantitative polymerase chain reaction studies of volunteers challenged with malaria sporozoites. J Infect Dis. 2005 Feb 15;191(4):619-26.

30. Douglas AD, Edwards NJ, Duncan CJ, et al. Comparison of modeling methods to determine liver-to-blood inocula and parasite multiplication rates during controlled human malaria infection. J Infect Dis. 2013 Jul 15;208(2):340-5.

31. Beaudoin RL, Strome CP, Mitchell F, et al. Plasmodium berghei: immunization of mice against the ANKA strain using the unaltered sporozoite as an antigen. Exp Parasitol. 1977 Jun;42(1):1-5.

*First demonstration, in mice, that sporozoite infection under chloroquine cover induces stage-specific immunity against homologous challenge.

32. Beaudoin RL, Strome CP, Tubergen TA, et al. Plasmodium berghei berghei: irradiated sporozoites of the ANKA strain as immunizing antigens in mice. Exp Parasitol. 1976 Jun;39(3):438-43.

33. Nussenzweig RS, Vanderberg J, Most H, et al. Protective immunity produced by the injection of x-irradiated sporozoites of plasmodium berghei. Nature. 1967 Oct $14 ; 216(5111): 160-2$.

34. Orjih AU, Cochrane AH, Nussenzweig RS. Comparative studies on the immunogenicity of infective and attenuated sporozoites of Plasmodium berghei. Trans R Soc Trop Med Hyg. 1982;76(1):57-61.

35. Sauerwein RW, Bijker EM, Richie TL. Empowering malaria vaccination by drug administration. Curr Opin Immunol. 2010 Jun;22(3):367-73. 
36. Accapezzato D, Visco V, Francavilla V, et al. Chloroquine enhances human CD8+ T cell responses against soluble antigens in vivo. J Exp Med. 2005 Sep 19;202(6):81728.

37. Friesen J, Silvie O, Putrianti ED, et al. Natural immunization against malaria: causal prophylaxis with antibiotics. Sci Transl Med. 2010 Jul 14;2(40):40ra49.

38. Ocana-Morgner C, Mota MM, Rodriguez A. Malaria blood stage suppression of liver stage immunity by dendritic cells. J Exp Med. 2003 Jan 20;197(2):143-51.

39. Lewis MD, Pfeil J, Heiss K, et al. CD8(+) T cells mediate robust stage-specific immunity to $P$. berghei under chemoprophylaxis and this protective environment is not downregulated by the presence of blood-stage infection. PLoS One. 2014;9(2):e88117.

40. Nganou-Makamdop K, van Gemert GJ, Arens T, et al. Long term protection after immunization with $P$. berghei sporozoites correlates with sustained IFNgamma responses of hepatic CD8+ memory T cells. PLoS One. 2012;7(5):e36508.

41. Brando C, Richardson JH, Murphy J, et al. Phenotypic characterization of Plasmodium berghei responsive $\mathrm{CD} 8+\mathrm{T}$ cells after immunization with live sporozoites under chloroquine cover. Malar J. 2014 Mar 12;13(1):92.

42. Spitalny G, Nussenzweig R. Effects of various routes of immunization and methods of parasite attenuation on the development of protection against sporozoite-induced rodent malaria. Mil Med. 1972;39:506-514.

43. Epstein JE, Tewari K, Lyke KE, et al. Live attenuated malaria vaccine designed to protect through hepatic $\mathrm{CD} 8(+) \mathrm{T}$ cell immunity. Science. 2011 Oct 28;334(6055):475-80. 
44. Nganou-Makamdop K, Ploemen I, Behet M, et al. Reduced Plasmodium berghei sporozoite liver load associates with low protective efficacy after intradermal immunization. Parasite Immunol. 2012 Dec;34(12):562-9.

45. Belnoue E, Costa FT, Frankenberg T, et al. Protective T cell immunity against malaria liver stage after vaccination with live sporozoites under chloroquine treatment. J Immunol. 2004 Feb 15;172(4):2487-95.

46. Sahu T, Lambert L, Herrod J, et al. Chloroquine neither eliminates liver stage parasites nor delays their development in a murine Chemoprophylaxis Vaccination model. Front Microbiol. 2015;6:283.

47. Inoue $\mathrm{M}$, Tang J, Miyakoda $\mathrm{M}$, et al. The species specificity of immunity generated by live whole organism immunisation with erythrocytic and pre-erythrocytic stages of rodent malaria parasites and implications for vaccine development. Int J Parasitol. 2012 Aug;42(9):859-70.

48. Doll KL, Butler NS, Harty JT. CD8 T cell independent immunity after single dose infection-treatment-vaccination (ITV) against Plasmodium yoelii. Vaccine. 2014 Jan $16 ; 32(4): 483-91$.

49. Nahrendorf W, Scholzen A, Sauerwein RW, et al. Cross-stage immunity for malaria vaccine development. Vaccine. 2015 Dec 22;33(52):7513-7.

50. Inoue M, Culleton RL. The intradermal route for inoculation of sporozoites of rodent malaria parasites for immunological studies. Parasite Immunol. 2011 Feb;33(2):13742.

51. Culleton RL, Inoue M, Reece SE, et al. Strain-specific immunity induced by immunization with pre-erythrocytic stages of Plasmodium chabaudi. Parasite Immunol. 2011 Jan;33(1):73-8. 
52. Nahrendorf W, Spence PJ, Tumwine I, et al. Blood-stage immunity to Plasmodium chabaudi malaria following chemoprophylaxis and sporozoite immunization. Elife. 2015 Feb 25;4:e05165.

53. Singh B, Daneshvar C. Human infections and detection of Plasmodium knowlesi. Clin Microbiol Rev. 2013 Apr;26(2):165-84.

54. Pichyangkul S, Spring MD, Yongvanitchit K, et al. Chemoprophylaxis with sporozoite immunization in $P$. knowlesi rhesus monkeys confers protection and elicits sporozoite-specific memory T cells in the liver. PLoS One. 2017;12(2):e0171826.

55. Roestenberg M, McCall M, Hopman J, et al. Protection against a malaria challenge by sporozoite inoculation. N Engl J Med. 2009 Jul 30;361(5):468-77.

**First demonstration in humans that $\boldsymbol{P}$. falciparum sporozoite infection (administered by mosquito bite) under chloroquine cover (CPS-CQ) induces complete sterile, protective immunity against sporozoite challenge.

56. Mordmuller B, Surat G, Lagler H, et al. Sterile protection against human malaria by chemoattenuated PfSPZ vaccine. Nature. 2017 Feb 23;542(7642):445-449.

** First demonstration that immunization by direct venous inoculation of purified, nonirradiated $P$. falciparum sporozoites under chloroquine cover (PFSPZ-CVac) induces complete, sterile protective immunity against sporozoite challenge.

57. Teirlinck AC, McCall MB, Roestenberg M, et al. Longevity and composition of cellular immune responses following experimental Plasmodium falciparum malaria infection in humans. PLoS Pathog. 2011 Dec;7(12):e1002389.

58. Roestenberg M, Bijker EM, Sim BKL, et al. Controlled human malaria infections by intradermal injection of cryopreserved Plasmodium falciparum sporozoites. Am J Trop Med Hyg. 2013 Jan;88(1):5-13. 
59. Roestenberg M, Teirlinck AC, McCall MB, et al. Long-term protection against malaria after experimental sporozoite inoculation: an open-label follow-up study. Lancet. 2011 May 21;377(9779):1770-6.

* Demonstration that the duration of the protective immunity induced by $P$. falciparum sporozoite infection under chloroquine cover (CPS-CQ) is at least two years.

60. Bijker EM, Bastiaens GJ, Teirlinck AC, et al. Protection against malaria after immunization by chloroquine prophylaxis and sporozoites is mediated by preerythrocytic immunity. PNAS. 2013;110(19):7862-7867.

61. Bijker EM, Schats R, Obiero JM, et al. Sporozoite immunization of human volunteers under mefloquine prophylaxis is safe, immunogenic and protective: a double-blind randomized controlled clinical trial. PLoS One. 2014;9(11):e112910.

62. Bijker EM, Teirlinck AC, Schats R, et al. Cytotoxic markers associate with protection against malaria in human volunteers immunized with Plasmodium falciparum sporozoites. J Infect Dis. 2014 Nov 15;210(10):1605-15.

63. Healy SA, Murphy SC, Hume JCC, et al. Chemoprophylaxis Vaccination: Phase I Study to Explore Stage-specific Immunity to Plasmodium falciparum in US Adults. Clin Infect Dis. 2020 Sep 12;71(6):1481-1490.

64. Bastiaens GJH, van Meer MPA, Scholzen A, et al. Safety, Immunogenicity, and Protective Efficacy of Intradermal Immunization with Aseptic, Purified, Cryopreserved Plasmodium falciparum Sporozoites in Volunteers Under Chloroquine Prophylaxis: A Randomized Controlled Trial. Am J Trop Med Hyg. 2016 Mar;94(3):663-673.

65. Schats R, Bijker EM, van Gemert GJ, et al. Heterologous Protection against Malaria after Immunization with Plasmodium falciparum Sporozoites. PLoS One. 2015;10(5):e0124243. 
66. Walk J, Reuling IJ, Behet MC, et al. Modest heterologous protection after Plasmodium falciparum sporozoite immunization: a double-blind randomized controlled clinical trial. BMC Med. 2017 Sep 13;15(1):168.

67. McCall MBB, Wammes LJ, Langenberg MCC, et al. Infectivity of Plasmodium falciparum sporozoites determines emerging parasitemia in infected volunteers. Sci Transl Med. 2017 Jun 21;9(395):eaag2490.

68. Moser KA, Drabek EF, Dwivedi A, et al. Strains used in whole organism Plasmodium falciparum vaccine trials differ in genome structure, sequence, and immunogenic potential. Genome Med. 2020 Jan 8;12(1):6.

69. Bijker EM, Schats R, Visser LG, et al. Ex vivo lymphocyte phenotyping during Plasmodium falciparum sporozoite immunization in humans. Parasite Immunol. 2015 Nov;37(11):590-8.

70. Tran TM, Bijker EM, Haks MC, et al. Whole-blood transcriptomic signatures induced during immunization by chloroquine prophylaxis and Plasmodium falciparum sporozoites. Sci Rep. 2019 Jun 10;9(1):8386.

71. Boyle MJ, Reiling L, Feng G, et al. Human antibodies fix complement to inhibit Plasmodium falciparum invasion of erythrocytes and are associated with protection against malaria. Immunity. 2015 Mar 17;42(3):580-90.

72. Behet MC, Kurtovic L, van Gemert GJ, et al. The Complement System Contributes to Functional Antibody-Mediated Responses Induced by Immunization with Plasmodium falciparum Malaria Sporozoites. Infect Immun. 2018 Jul;86(7):e0092017.

73. Felgner PL, Roestenberg M, Liang L, et al. Pre-erythrocytic antibody profiles induced by controlled human malaria infections in healthy volunteers under chloroquine prophylaxis. Sci Rep. 2013 Dec 19;3:3549. 
74. Peng K, Goh YS, Siau A, et al. Breadth of humoral response and antigenic targets of sporozoite-inhibitory antibodies associated with sterile protection induced by controlled human malaria infection. Cell Microbiol. 2016 Dec;18(12):1739-1750.

75. Obiero JM, Campo JJ, Scholzen A, et al. Antibody Biomarkers Associated with Sterile Protection Induced by Controlled Human Malaria Infection under Chloroquine Prophylaxis. mSphere. 2019 Feb 20;4(1):e00027-19.

76. Rts SCTP. Efficacy and safety of the RTS,S/AS01 malaria vaccine during 18 months after vaccination: a phase 3 randomized, controlled trial in children and young infants at 11 African sites. PLoS Med. 2014 Jul;11(7):e1001685.

77. Elliott SR, Kuns RD, Good MF. Heterologous immunity in the absence of variantspecific antibodies after exposure to subpatent infection with blood-stage malaria. Infect Immun. 2005 Apr;73(4):2478-85.

78. Cheesman S, Raza A, Carter R. Mixed strain infections and strain-specific protective immunity in the rodent malaria parasite Plasmodium chabaudi chabaudi in mice. Infect Immun. 2006 May;74(5):2996-3001.

79. Legorreta-Herrera M, Ventura-Ayala ML, Licona-Chavez RN, et al. Early treatment during a primary malaria infection modifies the development of cross immunity. Parasite Immunol. 2004 Jan;26(1):7-17.

80. Low LM, Ssemaganda A, Liu XQ, et al. Controlled Infection Immunization Using Delayed Death Drug Treatment Elicits Protective Immune Responses to Blood-Stage Malaria Parasites. Infect Immun. 2019 Jan;87(1):e00587-18.

** First demonstration that delayed-death apicoplast-targeting anti-malarial drugs administered concurrently with blood-stage parasites can induce protective immunity in mice. 
81. Dahl EL, Shock JL, Shenai BR, et al. Tetracyclines specifically target the apicoplast of the malaria parasite Plasmodium falciparum. Antimicrob Agents Chemother. 2006 Sep;50(9):3124-31.

82. Belnoue E, Voza T, Costa FT, et al. Vaccination with live Plasmodium yoelii blood stage parasites under chloroquine cover induces cross-stage immunity against malaria liver stage. J Immunol. 2008 Dec 15;181(12):8552-8.

83. Pombo DJ, Lawrence G, Hirunpetcharat $\mathrm{C}$, et al. Immunity to malaria after administration of ultra-low doses of red cells infected with Plasmodium falciparum. Lancet. 2002 Aug 24;360(9333):610-7.

** First demonstration that multiple, experimental, low dose P. falciparum blood-stage infections in humans can induce protective immunity mediated entirely by $T$ cells.

84. Edstein MD, Kotecka BM, Anderson KL, et al. Lengthy antimalarial activity of atovaquone in human plasma following atovaquone-proguanil administration. Antimicrob Agents Chemother. 2005 Oct;49(10):4421-2.

85. Herrera S, Solarte Y, Jordan-Villegas A, et al. Consistent safety and infectivity in sporozoite challenge model of Plasmodium vivax in malaria-naive human volunteers. Am J Trop Med Hyg. 2011 Feb;84(2 Suppl):4-11.

86. McCarthy JS, Griffin PM, Sekuloski S, et al. Experimentally induced blood-stage Plasmodium vivax infection in healthy volunteers. J Infect Dis. 2013 Nov $15 ; 208(10): 1688-94$

87. Bennett JW, Pybus BS, Yadava A, et al. Primaquine failure and cytochrome P-450 2D6 in Plasmodium vivax malaria. N Engl J Med. 2013 Oct 3;369(14):1381-2.

88. Aide P, Dobano C, Sacarlal J, et al. Four year immunogenicity of the RTS,S/AS02(A) malaria vaccine in Mozambican children during a phase IIb trial. Vaccine. 2011 Aug 11;29(35):6059-67. 
89. Camponovo F, Campo JJ, Le TQ, et al. Proteome-wide analysis of a malaria vaccine study reveals personalized humoral immune profiles in Tanzanian adults. Elife. 2020 Jul 14;9:e53080.

90. Sissoko MS, Healy SA, Katile A, et al. Safety and efficacy of PfSPZ Vaccine against Plasmodium falciparum via direct venous inoculation in healthy malaria-exposed adults in Mali: a randomised, double-blind phase 1 trial. Lancet Infect Dis. 2017 May;17(5):498-509. 
Table 1: Protective efficacy of different Plasmodium infection and drug cure regimens

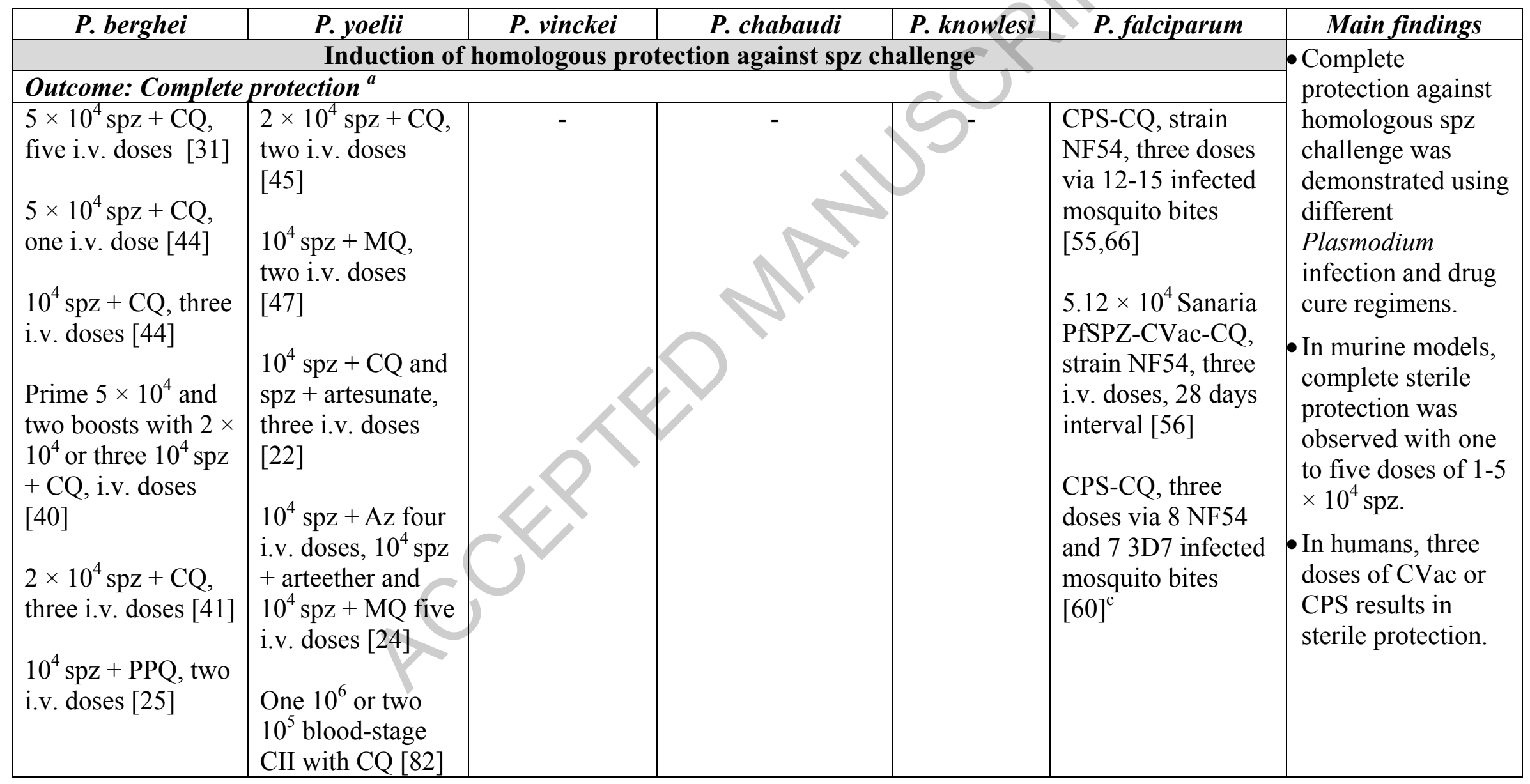




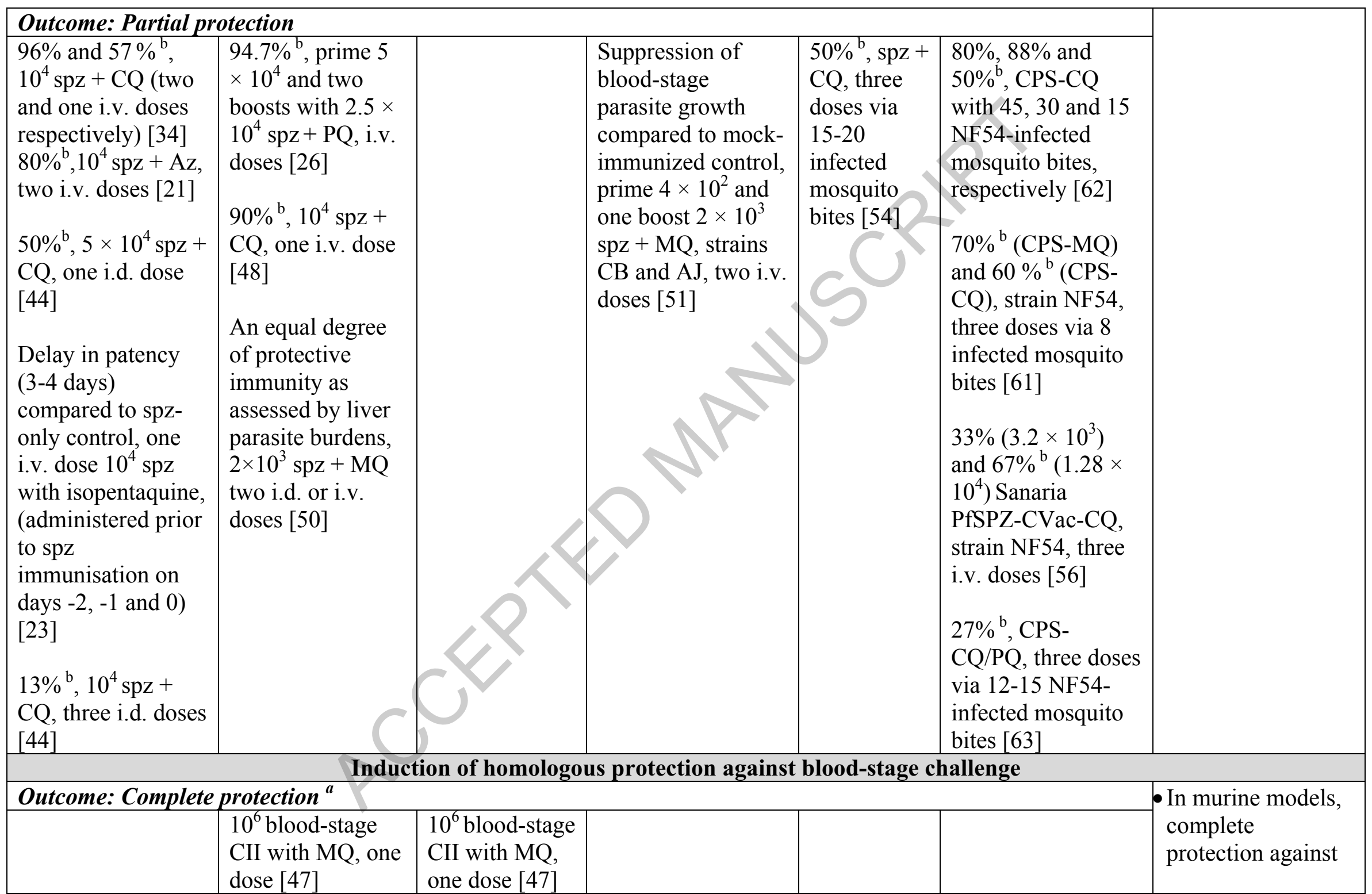




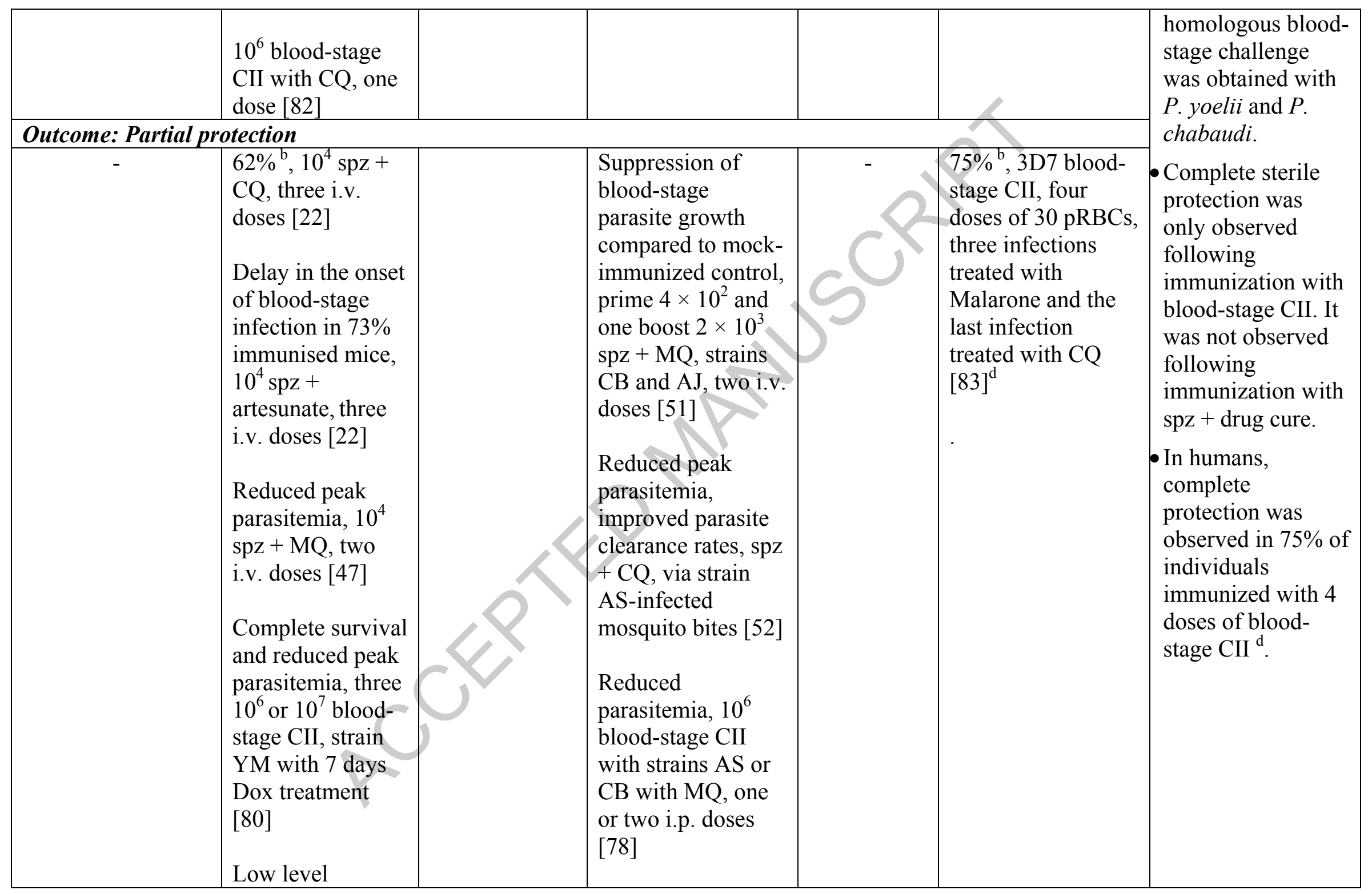




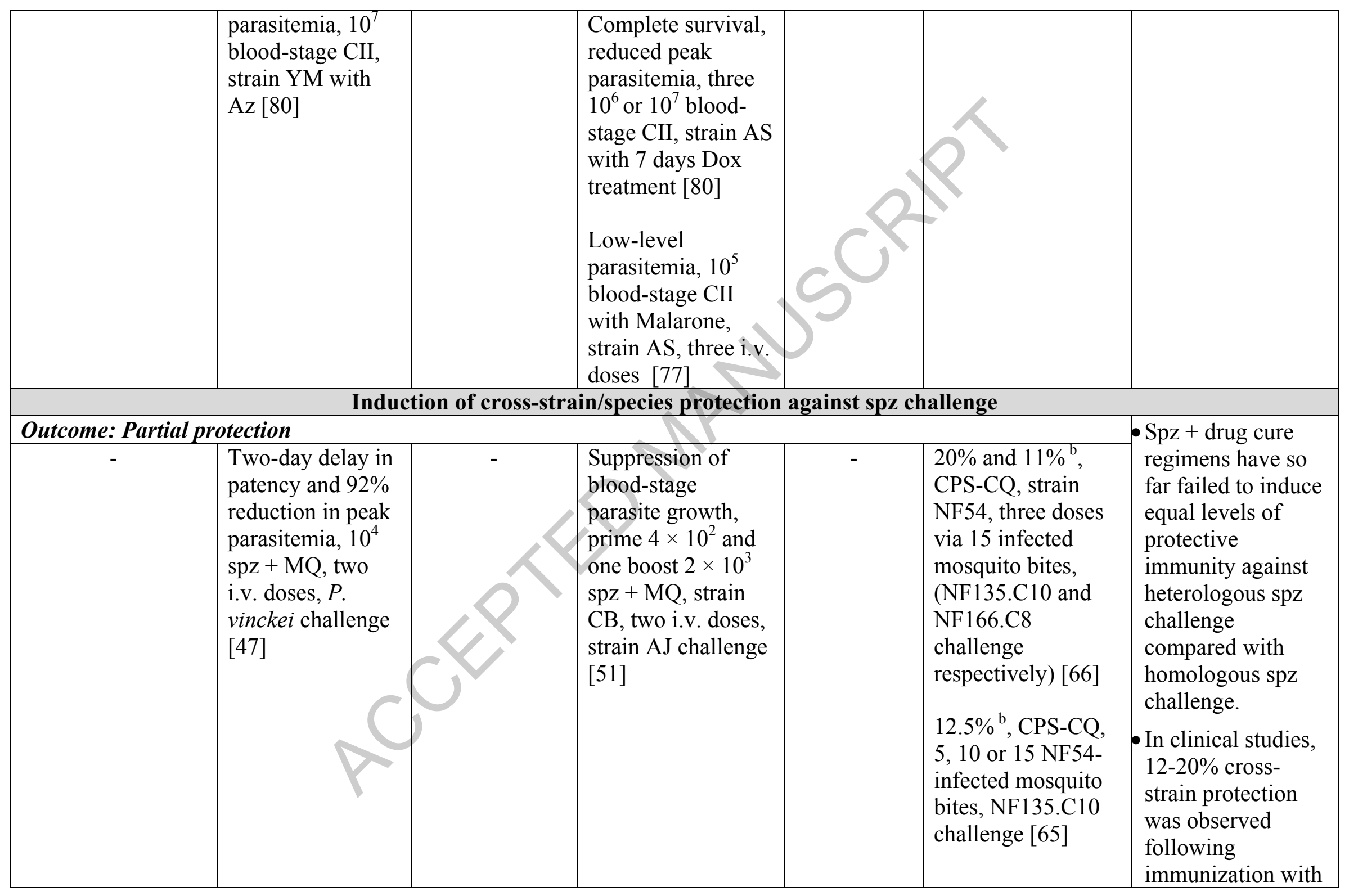




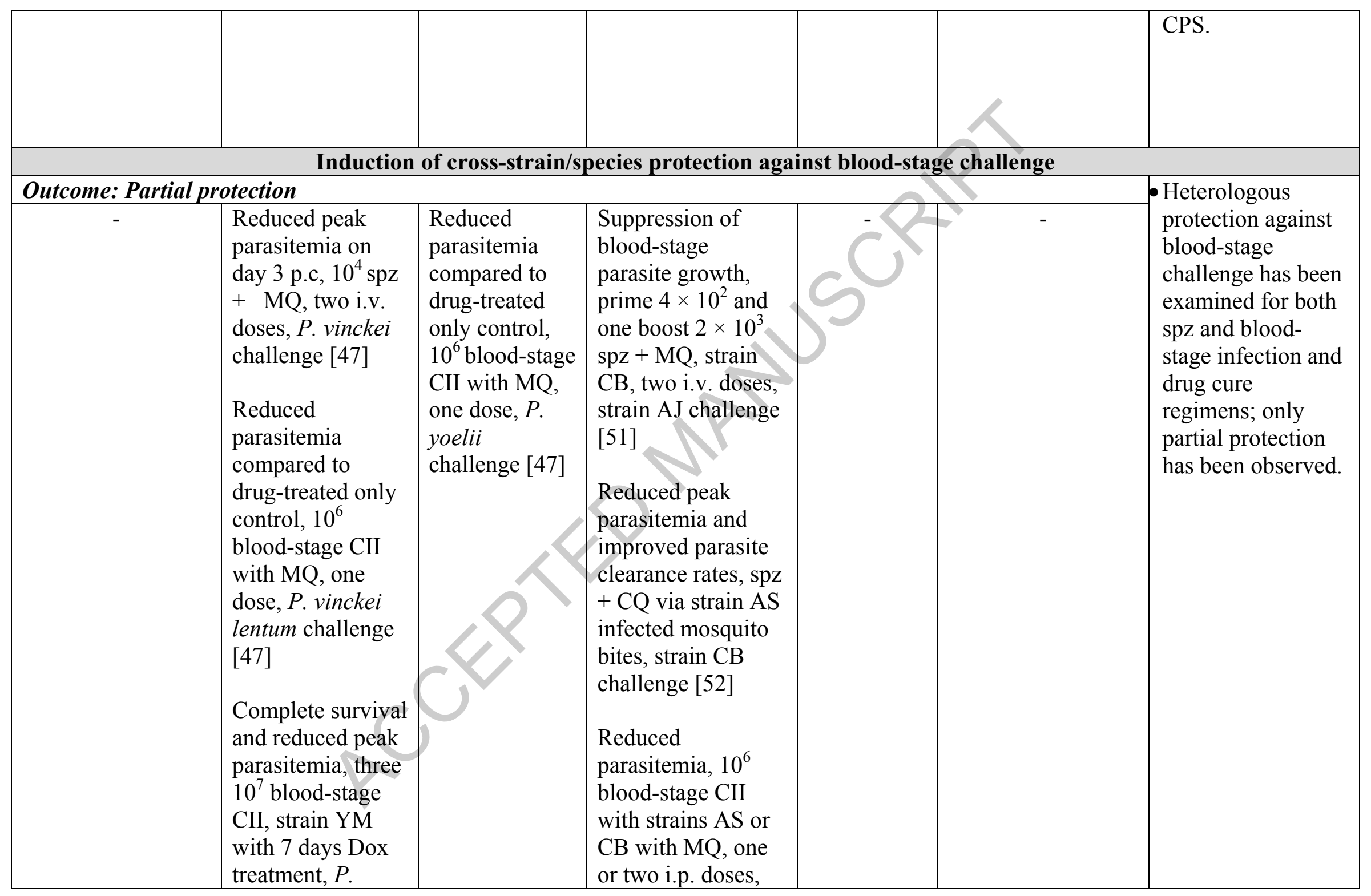




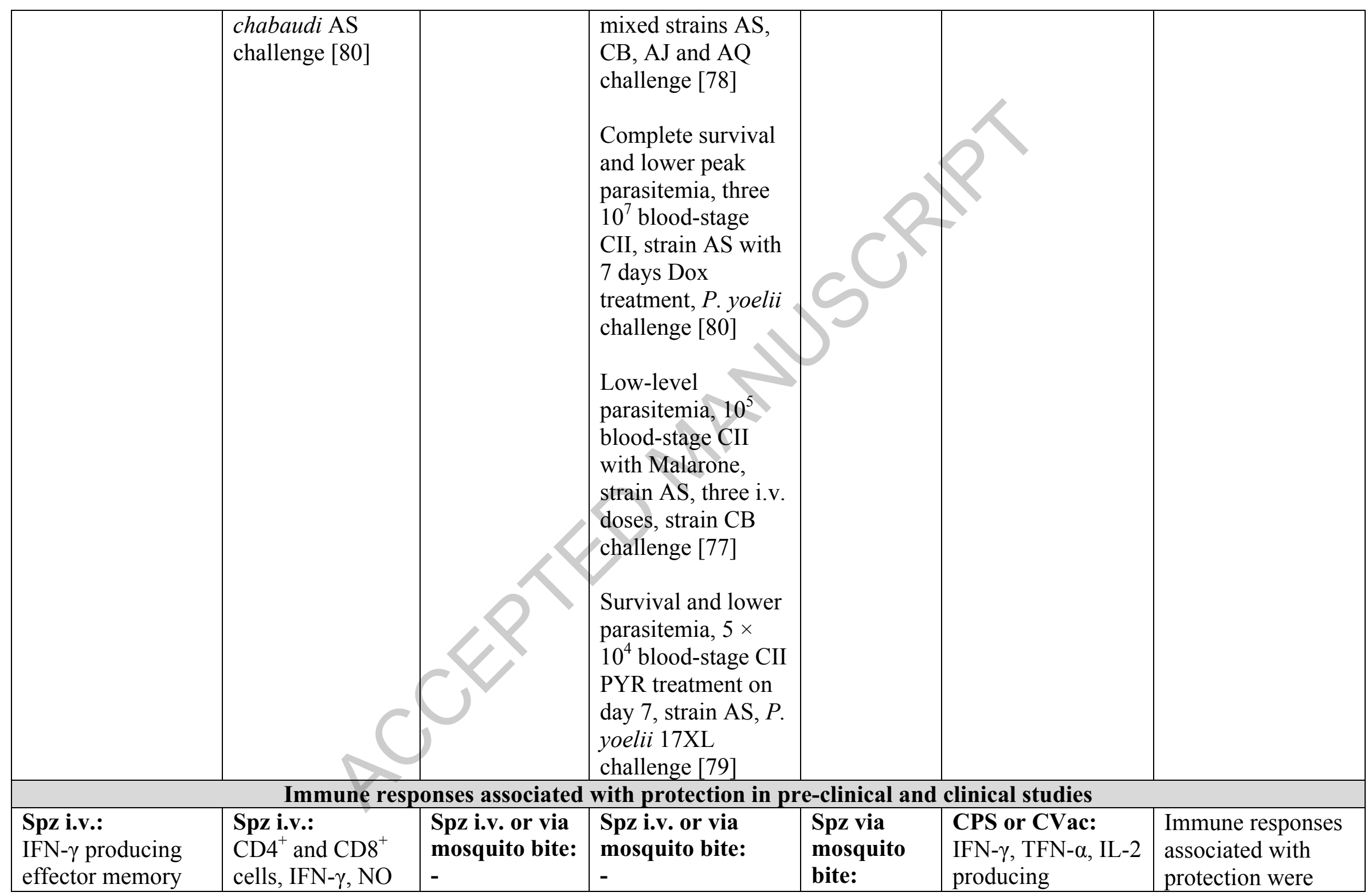




\begin{tabular}{|c|c|c|c|c|c|c|}
\hline $\begin{array}{l}\mathrm{CD}^{+} \mathrm{T} \text { cells [40] } \\
\text { Antibodies, CD } 8^{+} \\
\text {and } \mathrm{CD} 4^{+} \text {cells } \\
{[41]} \\
\text { Expansion of } \\
\text { effector memory } \\
\text { CD } 8^{+} \text {T-cells [25] } \\
\text { KLRG1 CD } 27^{\text {low }}, \\
\text { CD44 } \\
\text { CD62L }{ }^{\text {low }} \text { cells [41] } \\
\text { CD } 8^{+} \text {T effectors } \\
\text { cells, increased } \\
\text { IFN- } \gamma \text {, TNF- } \alpha, \text { and } \\
\text { IL-2 production } \\
{[23]} \\
\text { Blood-stage CII: } \\
-\end{array}$ & $\begin{array}{l}{[45]} \\
\mathrm{CD}^{+} \mathrm{T} \text { cells } \\
{[22,47]} \\
\text { Antibodies } \\
{[22,47,48]} \\
\text { Blood-stage CII: } \\
\text { Antibodies } \\
{[47,80,82]} \\
\mathrm{CD} 4^{+} \text {and CD8 } \\
\text { cells, IFN- } \gamma, \mathrm{NO} \\
{[82]}\end{array}$ & $\begin{array}{l}\text { Blood-stage } \\
\text { CII: } \\
\text { Antibodies } \\
{[47]}\end{array}$ & $\begin{array}{l}\text { Blood-stage CII: } \\
\text { Antibodies to } \\
\text { conserved antigens, } \\
\text { Th1 response (IFN- } \\
\gamma^{+} \mathrm{CD}^{+} \\
\text {lymphocytes) [77] } \\
\text { Th2 response with } \\
\text { higher IL-4 and IL- } \\
10 \text { levels and lower } \\
\text { IFN- } \gamma \text { expression } \\
\text { [79] } \\
\text { Th1 cytokine } \\
\text { response (IFN- } \gamma, \\
\text { TNF- } \alpha, \text { IL-2) [80] }\end{array}$ & $\begin{array}{l}\text { Antibodies, } \\
\text { memory T- } \\
\text { cells } \\
\text { expressing } \\
\text { CCR5 and } \\
\text { CXCR6 and } \\
\text { CD69 [54] } \\
\text { Blood-stage } \\
\text { CII: }\end{array}$ & $\begin{array}{l}\text { pluripotent effector } \\
\text { memory T-cell } \\
\text { [55,59] } \\
\text { Polyfunctional } \\
\text { IFN- } \gamma \text {, IL-2 and } \\
\text { TNF- } \alpha \text { producing } \\
\text { CD4 }{ }^{+} \text {T-cells [56] } \\
\text { IFN- } \gamma \text { and } \\
\text { granzyme B T-cell } \\
\text { responses [66] } \\
\text { T-cell memory } \\
\text { response [70] } \\
\text { Cytotoxic markers } \\
\text { (CD4 }{ }^{+} \text {T-cells } \\
\text { expressing CD107a } \\
\text { and granzyme B- } \\
\text { producing CD } 8^{+} \text {T- } \\
\text { cells) [62] } \\
\gamma \delta \text { T-cells, Th1 and } \\
\text { cytotoxic responses } \\
{[61,69]} \\
\text { Complement-fixing } \\
\text { IgM and IgG1 } \\
\text { antibodies [72] } \\
\text { Antibodies [61, 66, }\end{array}$ & $\begin{array}{l}\text { variable between } \\
\text { studies. }\end{array}$ \\
\hline
\end{tabular}




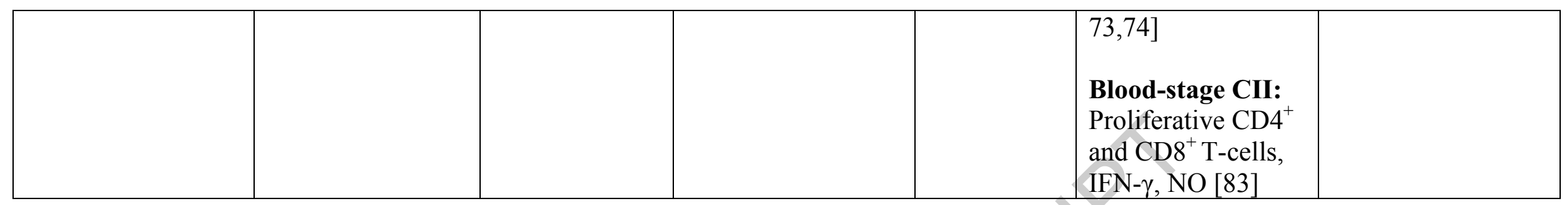

Abbreviations: Az: azithromycin, CII: controlled infection immunization, CPS: chemoprophylaxis and sporozoite immunization, CQ: chloroquine, CVac: chemoprophylaxis vaccination, Dox: doxycycline, IFN- $\gamma$ : interferon-gamma, IL: interleukin, KLRG1: killer-cell 
lectin-like receptor G1, MQ: mefloquine, NO: nitric oxide, PfSPZ-CVac: Plasmodium falciparum sporozoites chemoprophylaxis vaccination, p.c.: post-challenge, PPQ: piperaquine, PQ: primaquine, PYR: pyrimethamine, spz: sporozoites, TNF- $\alpha$ : Tumor necrosis factor-alpha. ${ }^{a}$ Complete protection in immunized group represents total absence of bloodstage parasitemia following challenge. For partial protection: ${ }^{b} \%$ protection in immunized group represents number protected against development of blood-stage parasitemia following challenge / number challenged; ${ }^{c}$ Low level blood-stage parasitemia was detected by qPCR retrospectively in one of the five participants on the day of presumptive drug treatment (Day 21 post challenge)[60]; ${ }^{\mathrm{d}}$ residual anti-malarial drug may have contributed to this protection [84]. 


\section{Figure Legends}

Figure 1: The life-cycle of Plasmodium spp. parasites.

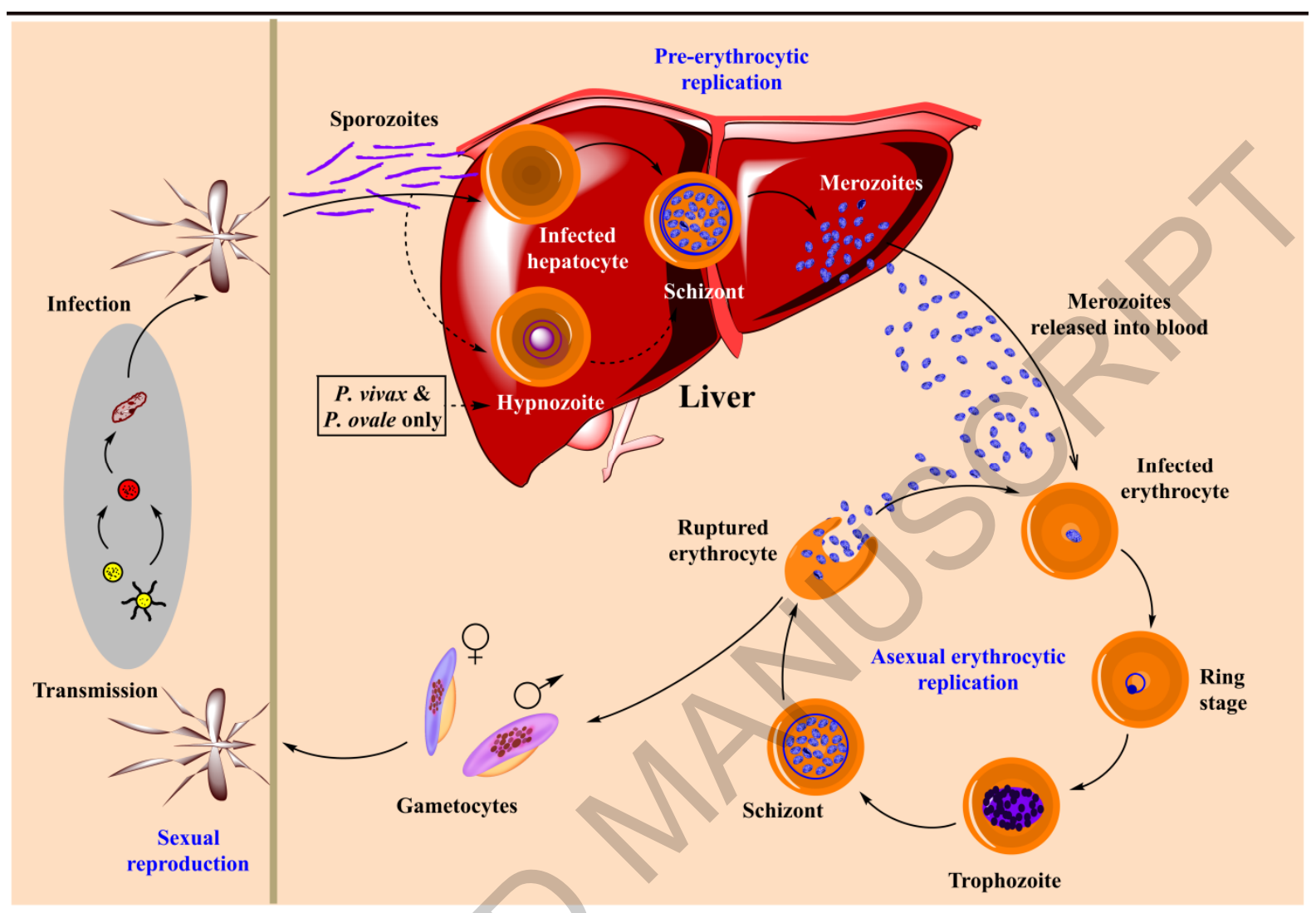

Figure 2: Plasmodium infection and drug cure as a vaccine approach to induce protective immunity. A) Schematic of Plasmodium infection and drug cure vaccine regimens; B) Stagespecificity of anti-malarial drugs used in the sporozoite infection and drug cure regimens for immunization, CPS and CVac. Drugs targeting liver-stage parasites arrest parasites in the liver, impacting on the release of parasites into the bloodstream while drugs targeting bloodstage parasites prevent the establishment of a blood-stage infection by preventing the development of merozoites and their subsequent release into the bloodstream. Abbreviations: Az, azithromycin; CPS, chemoprophylaxis and sporozoite immunization; CQ, chloroquine; CVac, chemoprophylaxis vaccination; $\mathrm{MQ}$, mefloquine; PPQ: piperaquine; PQ, primaquine, PYR, pyrimethamine; i.d., intradermal; i.v., intravenous. 


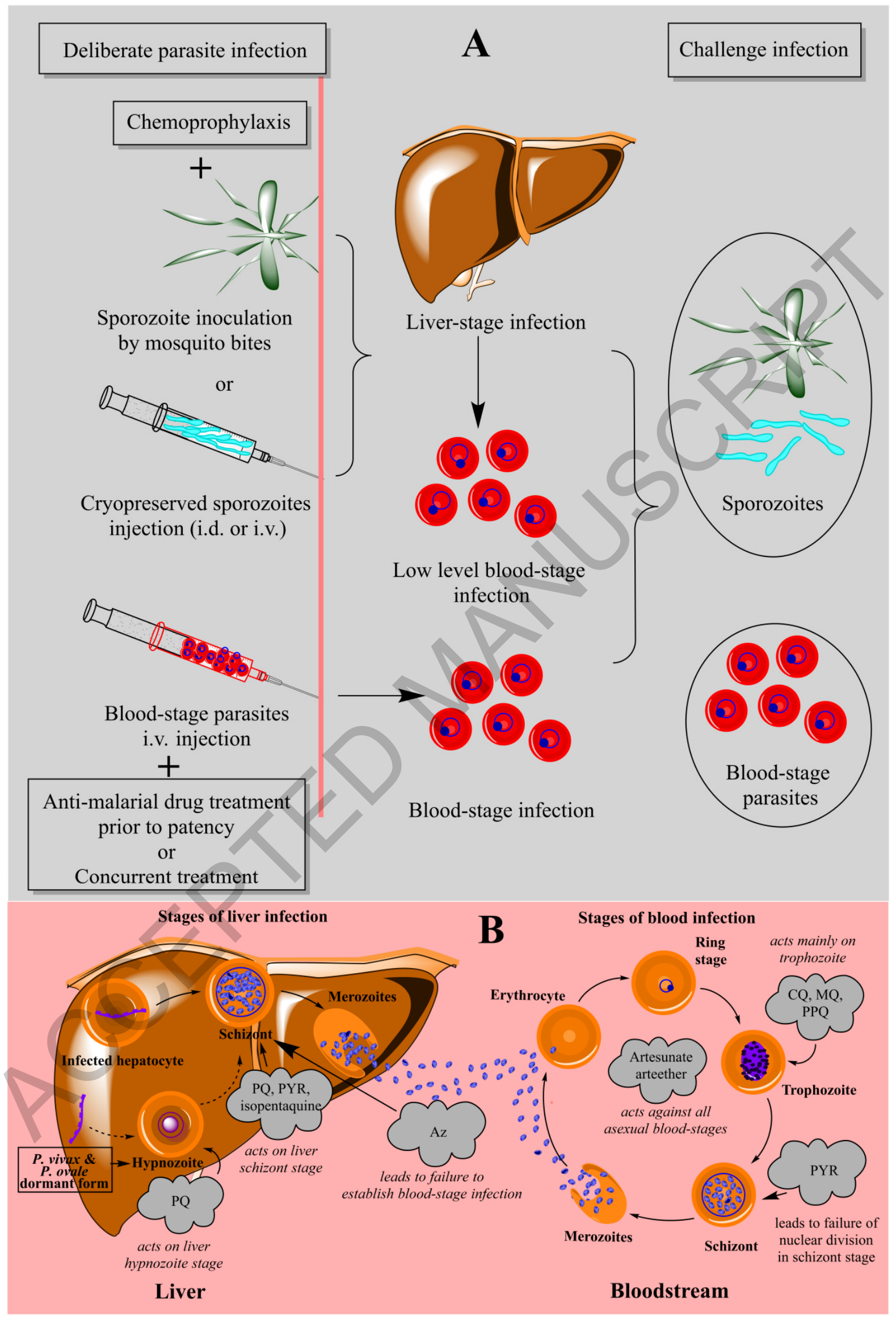

